\title{
Sistemática, taxonomía y domesticación de alpacas y llamas: nueva evidencia cromosómica y molecular
}

\author{
Systematics, taxonomy and domestication of alpaca and llama: new chromosomal \\ and molecular evidence
}
JUAN C. MARÍN ${ }^{1,8},{ }^{*}$, BEATRIZ ZAPATA², BENITO A. GONZÁLEZ ${ }^{3}$, CRISTIAN BONACIC ${ }^{4}$ JANE C. WHEELER ${ }^{5}$, CIARA CASEY ${ }^{6}$, MICHAEL W. BRUFORD ${ }^{6}$, R. EDUARDO PALMA ${ }^{7}$, ELIE POULIN $^{7,8}$, M. ANGÉLICA ALLIENDE ${ }^{9} \&$ ÁNGEL E. SPOTORNO ${ }^{1}$

\author{
${ }^{1}$ Laboratorio de Genómica y Biodiversidad, Departamento de Ciencias Básicas,Facultad de Ciencias, \\ Universidad del Bío-Bío, Casilla 447, Chillán, Chile \\ ${ }^{2}$ Facultad de Ciencias Veterinarias y Pecuarias, Universidad de Chile \\ ${ }^{3}$ Facultad de Ciencias Forestales, Universidad de Chile \\ ${ }^{4}$ Facultad de Agronomía e Ingeniería Forestal, Pontificia Universidad Católica de Chile \\ ${ }^{5}$ CONOPA, Coordinadora de Investigación y Desarrollo de Camélidos Sudamericanos, Lima, Perú \\ ${ }^{6}$ Cardiff School of Biosciences, Cardiff University, United Kingdom \\ ${ }^{7}$ Departament of Biological Science, University of Lincoln, United Kingdom \\ ${ }^{8}$ Centro de Estudios Avanzados de Ecología y Biodiversidad y Departamento de Ecología, \\ Facultad de Ciencias Biológicas, Pontificia Universidad Católica de Chile \\ ${ }^{9}$ Instituto de Ecología y Biodiversidad, Laboratorio de Ecología Molecular, Departamento de Ciencias Ecológicas, \\ Facultad de Ciencias, Universidad de Chile \\ ${ }^{10}$ Laboratorio de Citogenética, INTA, Universidad de Chile \\ ${ }^{11}$ Laboratorio de Genómica Evolutiva de Mamíferos, ICBM, Facultad de Medicina, Universidad de Chile \\ *e-mail para correspondencia: jmarin@med.uchile.cl
}

\begin{abstract}
RESUMEN
Existen cuatro especies de camélidos sudamericanos, dos de ellos silvestres, guanaco (Lama guanicoe) y vicuña (Vicugna vicugna), y dos formas domésticas, alpaca (Lama pacos) y llama (Lama glama), cuyo origen ha sido objeto de debate. En el presente estudio la variación en el patrón de bandas G de los cromosomas de llamas y alpacas y la secuencia de dos genes mitocondriales han sido usados para estudiar el origen y la clasificación de llamas y alpacas. Patrones de bandas cromosómicas similares fueron observados en las cuatro especies de Lamini, incluso similares a los descritos para camello, Camelus bactrianus. Sin embargo, se encontraron finas y consistentes diferencias en los brazos cortos del cromosoma 1, permitiendo separar a camellos, guanacos y llamas, de las de vicuñas y alpacas. Este patrón fue consistente incluso en un híbrido guanaco x alpaca. Relaciones equivalentes fueron encontradas en las secuencias completas del gen para citocromo $b$, así como en el árbol de expansión mínima de las secuencias parciales de la región control, agrupando a guanacos con llamas y a vicuñas con alpacas. Los análisis filogenéticos mostraron a $V$. vicugna y a L. guanicoe como grupos recíprocamente monofiléticos. El análisis de las secuencias de ambos genes mostró dos clados entre las vicuñas, concordantes con las subespecies reconocidas para esta especie, pero los resultados obtenidos para guanacos no reflejaron la existencia de las cuatro subespecies previamente propuestas. El análisis combinado de variaciones cromosómicas y moleculares demostraron una alta similitud genética entre alpacas y vicuñas, así como entre llamas y guanacos. Aunque se revela hibridización direccional, nuestros resultados apoyan fuertemente la hipótesis de que la llama se deriva de L. guanicoe, y la alpaca de V. vicugna, apoyando la reclasificación de la alpaca como $V$. pacos.
\end{abstract}

Palabras clave: Camelidae, Lama pacos, Vicugna pacos, Lama glama, cromosomas, citocromo b, d-loop.

\begin{abstract}
Four camelid species exist in South America: two wild, the guanaco (Lama guanicoe) and the vicuña (Vicugna vicugna), and two domestic, the alpaca (Lama pacos) and the llama (Lama glama). However, the origin of the domestic species has been a matter of debate. In the present study, variations in chromosome G banding patterns and in two mitochondrial gene sequences have been used to study the origin and classification of the llama and alpaca.-Similar patterns in chromosome $\mathrm{G}$ band structure were observed in all four Lamini species, and these in turn were similar to the bands described for camels, Camelus bactrianus. However, fine and consistent differences were found in the short arms of chromosome 1, separating camels, guanacos and llamas from vicuñas and alpacas. This pattern was consistent even in a hybrid guanaco $\mathrm{x}$ alpaca. Equivalent relationship
\end{abstract}


showed the complete cytochrome $b$ gene sequences, and the minimum expansion tree of the partial control region sequence, grouping guanaco with llama and vicuña with alpaca. Phylogenetic analyses showed $V$. vicugna and L. guanicoe as monophyletic groups. Analysis of both gene sequences revealed two clades within vicuña, concordant with the two described subspecies, but the results for guanaco did not confirm existence of the four previously proposed subspecies. The combined analysis of chromosomal and molecular variation showed close genetic similarity between alpacas and vicuñas, as well as between llamas and guanacos. Although directional hybridization was revealed, our results strongly support the hypothesis that the llama would have derived from L. guanicoe and the alpaca from $V$. vicugna, supporting reclassification as $V$. pacos.

Key words: Camelidae, Lama pacos, Vicugna pacos, Lama glama, chromosome, cytochrome $b$, d-loop.

\section{INTRODUCCIÓN}

Los camélidos están actualmente representados en Asia y África, así como en Sudamérica (Nowack 1991). Esta dicotomía biogeográfica es concordante con la taxonomía del grupo, pues los representantes de ambas regiones se clasifican en las tribus Camelini y Lamini, respectivamente. Cuatro especies de camélidos habitan hoy en Sudamérica, dos de ellas silvestres: el guanaco (Lama guanicoe Müller 1776) y la vicuña (Vicugna vicugna Molina 1782), mientras que las formas domésticas corresponden a la llama (Lama glama Linnaeus, 1758) y la alpaca (Lama pacos Linneaus 1758).

El guanaco es el artiodáctilo silvestre más grande de Sudamérica, con una amplia distribución en ambientes áridos y semiáridos de Sudamérica, desde el nivel del mar hasta los $5.200 \mathrm{~m}$ de altitud (Wheeler 1991). Cuatro subespecies se reconocen en L. guanicoe caracterizadas por sutiles diferencias morfológicas distribuidas a uno y otro lado de los Andes (Wheeler 1995, González et al. 2006), desde la Reserva Nacional de Calipuy ( $\left.8^{\circ} \mathrm{S}\right)$, en el centro-norte del Perú, hasta la isla Navarino en el extremo sur de Chile $\left(55^{\circ} \mathrm{S}\right)$. De ellos, el taxón más septentrional, L. g. cacsilensis (Lönnberg 1913) habita el centro y sur del Perú y norte de Chile entre 8 y $20^{\circ} \mathrm{S}$; más al sur, L. g. huanacus (Molina 1782) se distribuye en la zona centro norte de Chile entre $22^{\circ}$ y $28^{\circ} \mathrm{S}$. Sobre el flanco oriental de la cordillera de los Andes, L. g. voglii (Krumbiegel 1944) ocurre en el norte de Argentina y el Chaco de Bolivia y Paraguay entre $21^{\circ}$ y $32^{\circ} \mathrm{S}$. Finalmente la forma más austral, $L$. g. guanicoe (Müller 1776) habita en el rango desde los $32^{\circ} \mathrm{S}$ hacia el sur, cubriendo gran parte de la Patagonia de Argentina y Chile, e islas de Tierra del Fuego y Navarino (Krumbiegel 1944, Franklin 1982, Wheeler 1995).

Por otro lado, dos subespecies se reconocen en vicuñas basadas en caracteres morfológicos que incluyen diferencias de tamaño, coloración, forma del pelaje y longitud de los molares. Estas características, junto con su distribución geográfica, separan a $V . v$. mensalis (Thomas 1917) de V. v. vicugna (Molina 1782). V. v. mensalis se distribuye en Perú, Bolivia y Chile entre los 9 y $18^{\circ} \mathrm{S}$ y se caracteriza por ser una forma más grácil (45 cm de alzada) de fibra color canela oscura, presencia de un mechón de fibra larga y blanca en el pecho y menor longitud de la serie molar $(57 \mathrm{~mm})$ si se compara con V. v. Vicugna (Wheeler 1995). Esta última, en cambio, posee una fibra más clara, mayor alzada $(70 \mathrm{~cm})$ y una longitud de la serie molariforme de $90 \mathrm{~mm}$, cuya distribución incluye Bolivia, Chile y Argentina entre los 18 y $29^{\circ} \mathrm{S}$ (Wheeler 1995). Sin embargo, y a pesar de las diferencias morfológicas y de distribución de ambos taxa, algunos autores no aceptan la existencia de $V$. v. mensalis (Osgood 1943, Gilmore 1950).

Cuando en 1758 Linneaus describe por primera vez las dos formas domésticas de camélidos del Nuevo Mundo como Camelus glama "Camelus peruvianus Glama dictus" (llama) y Camelus pacos "Camelus peruvianus laniger Pacos dictus", ubicó estas nuevas especies junto a camellos y dromedarios en el género Camelus. Las formas silvestres serían luego nombradas Camelus guanicoe y Camelus vicugna (Wheeler 1995). Tempranamente en 1775 , Frisch propuso que las cuatro especies de camélidos sudamericanos fuesen indexadas en el género Lama. Sin embargo, no fue sino hasta 1924 que Miller separó la vicuña en el género Vicugna, sobre la base del crecimiento continuo de incisivos. Sin embargo, Miller no incluyó incisivos de alpaca en su estudio y por tanto no se percató de la similitud entre estos y los incisivos de la vicuña. Es debido a esta omisión que desde entonces ambas formas domésticas y el guanaco comparten el género Lama (Wheeler 2005). En la actualidad, la Comisión 
Internacional de Nomenclatura Zoológica recomienda conservar los nombres de las especies silvestres de las cuales habrían derivado sus formas domésticas, tal como lo propusieron Gentry, Clutton-Brock y Groves (Opinión 2027, marzo 2003). Con excepción de 17 casos (una mariposa, un pez y quince mamíferos), la mayoría de los progenitores silvestres y sus formas domésticas derivadas, comparten el mismo nombre. Uno de estos mamíferos sobre los cuales se produce esta excepción es la alpaca.

Por otra parte, el origen de llamas y alpacas ha sido históricamente material de controversia (Wheeler 2005) debido principalmente a la alta tasa de hibridización entre estas formas y a dificultades en la interpretación de los restos zooarqueológicos (Kadwell et al. 2001). La alpaca ha sido descrita como descendiente del guanaco, la vicuña y la llama. Evidencia basada en la morfología dentaria, sin embargo, sostiene que llamas y alpacas se habrían originado a partir de guanacos y vicuñas, respectivamente (Wheeler 1984). Una tercera hipótesis, actualmente desacreditada, sostiene que guanacos y vicuñas nunca fueron sometidos a domesticación, y que tanto alpaca como llama habrían especiado a partir de una forma ancestral ya extinta (Cabrera 1932). Alternativamente, otros estudios sustentan la hipótesis que la alpaca sería un híbrido entre llama y vicuña (Hemmer 1990). En cualquier caso, el parentesco cercano entre llama y guanaco no ha sido cuestionado.

La morfología de incisivos, características del pelaje, patrones de conducta, así como análisis moleculares, han contribuido también a la controversia respecto del origen de las formas domésticas. Al comparar la morfología de incisivos de restos arqueológicos de la puna peruana, se detectó similitudes entre alpacas y vicuñas, lo que permitió concluir que las alpacas eran formas domésticas de vicuñas (Wheeler 1984, 1995). Material faunístico de tales sitios indicarían que durante los inicios de la ocupación humana, 12.000 a 7.500 años atrás, tanto guanacos y vicuñas, como cérvidos (Hippocamelus antisensis, taruca o huemul del norte), eran cazados persistentemente en la región (Wheeler 1984, 1995, Wing 1986). En períodos posteriores (9.000 a 7.000 años), se registra un notorio aumento de restos de camélidos, y ya desde 6.000 a 5.500 años atrás se detecta el surgimiento de las primeras alpacas y llamas en asentamientos humanos (Wheeler 1995).

A nivel cromosómico, estudios dirigidos a la determinación del origen de la alpaca han producido resultados contradictorios. Los camélidos del Viejo y Nuevo Mundo presentan un cariotipo muy conservado, $2 \mathrm{n}=74$ (Taylor et al. 1968; Koulischer et al. 1971), con patrones de bandas $\mathrm{G}$ y C aparentemente muy similares (Bunch et al. 1985), siendo capaces de cruzarse y producir descendencia fértil bajo influencia humana. Algunos intentos por establecer relaciones de parentesco usando métodos inmunogenéticos (Penedo et al. 1988) han fallado también. Sin embargo, la comparación de perfiles de hibridización de DNA satélite mostró un patrón indistinguible entre guanacos y llamas pero diferente del de vicuñas. Un patrón intermedio entre guanacos y vicuñas habría sido detectado en alpacas (Vidal-Rioja et al. 1994). Usando la secuencia parcial del gen para citocromo $b$ de formas domésticas y silvestres de camélidos sudamericanos, Stanley et al. (1994) demostraron que guanacos y vicuñas son parte de diferentes clados. En un estudio más reciente, usando la secuencia parcial del gen para citocromo $b(900$ pb), Palma et al. (2001) evaluaron el estatus taxonómico de 3 subespecies de guanaco (Lama guanicoe guanicoe, L. g. cacsilensis y L. g. huanacus) y ambas subespecies de vicuñas. Este estudio reforzó la monofilia de L. guanicoe, sin embargo, mostró a $V$. vicugna como grupo polifilético, dejando a la llama como el taxón hermano del guanaco, y la alpaca de la vicuña. También, Kadwell et al. (2001) publicaron los resultados del análisis de la secuencia parcial del gen citocromo $b$ y de cuatro microsatélites en una muestra de más de 700 llamas, alpacas, vicuñas y guanacos a nivel transandino y demostraron que la alpaca es la forma doméstica de la vicuña y la llama del guanaco. De esta manera, se fortaleció la hipótesis proveniente de evidencias arqueozoológicas de que la llama podría haber derivado de la domesticación de una o varias poblaciones de guanaco, y la alpaca de una o varias poblaciones de vicuñas (Wheeler 1984, 1995). A nivel molecular también, secuencias parciales del gen para citocromo $b$ del ADNmt (Stanley et al. 1994, Wheeler et al. 2006) y la disposición de ADN satélite (VidalRioja 1994) han establecido la ocurrencia de hibridación en el pasado. 
Con el objeto de dilucidar las relaciones filogenéticas de los camélidos sudamericanos, y particularmente el origen de las formas domésticas, analizamos el patrón de bandeo $\mathrm{G}$ cromosómico, las secuencias completa del gen para citocromo $b$ así como la del Dominio Hipervariable I de la Región Control del ADNmt en los cuatro taxa. Considerando muestras de más de 50 localidades y de todo el rango de distribución de guanacos y vicuñas, así como de representantes de las distintas variedades domésticas de Perú y Chile, entregamos nuevas evidencias sobre el origen, domesticación y posición taxonómica de llamas y alpacas.

\section{MATERIALES Y MÉTODOS}

\section{Análisis cromosómico}

Se obtuvo una muestra de $10 \mathrm{~mL}$ de sangre periférica con tubos venoject o vacutainer heparinizados usando agujas estériles desechables $21 \mathrm{G}$, para cada uno de los siguientes animales: 22 L. guanicoe guanicoe procedentes de la XII Región de Magallanes mantenidos en la Estación Experimental de la Pontificia Universidad Católica de Chile, Región Metropolitana; nueve L. glama, tres procedentes del criadero La Disputada de Las Condes, RM, dos de la Estación Experimental INIA-Kampenaike, Magallanes, y cuatro del criadero de la Estación Experimental INIAHidango, VI Región; 10 V. v. mensalis, de las cuales cinco fueron capturadas en el Salar de Surire, I Región, y cinco del mismo origen, mantenidas en el Jardín Zoológico del Parque Metropolitano, RM; y 10 L. pacos, tres procedentes de la Estación Experimental de la Pontificia Universidad Católica de Chile, RM, dos de la Facultad de Ciencias Veterinarias, Universidad de Chile, Región Metropolitana, dos del criadero de la Estación Experimental INIA-Hidango, VI Región, y tres procedentes del criadero La Disputada de Las Condes, Región Metropolitana. Adicionalmente, se estudió material proveniente de un híbrido guanaco x alpaca proveniente de un criadero de los alrededores de Vallenar, III Región, cuya madre fue un guanaco traído desde la cordillera de la zona. Los sobrenadantes de las muestras de sangre centrifugadas a $800 \mathrm{rpm}$ se sembraron el mismo día en $5 \mathrm{~mL}$ de medio de cultivo RPMI 1640, con L-glutamina, suero bovino fetal y fitohemaglutinina, cultivados por $72 \mathrm{~h}$, cosechados y tratados según protocolos estandarizados para bandas G y C (Zapata 1999). Para el conteo cromosómico se usó un mínimo de 30 placas metafásicas seleccionadas por calidad; los cromosomas fueron ordenados en orden decreciente de tamaño, medidos en 10 células por individuo, y sus promedios usados para confeccionar los correspondientes ideogramas de cada especie. La nomenclatura para bandas $G$ sigue las recomendaciones formuladas para animales domésticos (Ford et al. 1980).

\section{Análisis moleculares}

Un total de 172 muestras de guanacos y 189 de vicuñas fueron obtenidas en terreno a través de tres métodos alternativos: (i) de ejemplares vivos a través de la inmovilización química de animales juveniles o adultos con la aplicación de 3,5 a $8 \mathrm{mg} \mathrm{kg}^{-1}$ de Tiletamine-Zolazepam (modificado de Sarno et al. 1996), o con la captura masiva de animales adultos ("chaku") aprovechando manejos de esquila de fibra. De cada animal se extrajo $10 \mathrm{~mL}$ de sangre venosa a través de jeringas vacutainer con EDTA mantenidos a $4{ }^{\circ} \mathrm{C}$; (ii) de animales muertos encontrados en terreno, extrayendo una muestra de músculo o piel que fue almacenado en etanol $70^{\circ}$ a temperatura ambiente; (iii) de fecas frescas de animales individualizados en terreno, las que fueron mantenidas a temperatura ambiente en etanol absoluto. De ejemplares domésticos se extrajo $10 \mathrm{~mL}$ de sangre a 30 llamas y 32 alpacas mantenidas en cautiverio en criaderos de Chile y Perú, fueron colectados a través de jeringas vacutainer con EDTA (Tabla 1).

El ADN genómico de estas muestras, por su distinta naturaleza, fueron extraídos a través de cuatro métodos: (i) a partir de $10 \mathrm{~mL}$ de sangre, usando una modificación de la técnica de Lahiri \& Nurnberger (1991); (ii) desde muestras de piel o pelos se extrajo ADN usando CHELEX 5 $\%$ (Walsh 1991) y proteinasa K; (iii) a partir de hígado o músculo preservado en alcohol $70^{\circ}$, se obtuvo ADN usando Cloroformo-fenol y precipitación en Etanol, previo homogeneizado en STE, SDS y proteinasa K (Medrano et al. 1990); finalmente (iv) a partir de fecas se usó el kit comercial QIAGEN QIAamp. DNA Stool Mini. 


\section{TABLA 1}

Listado de especímenes de camélidos sudamericanos reunidos para la realización de esta publicación. Se indica el tipo de muestra (S: sangre, F: heces, M: músculo, MO: médula ósea, H: hígado), localidad, coordenadas geográficas y número de individuos (n)

List of specimens of South American camelids sampled. Type of sample (S: blood, F: faeces; M: muscle; MO: bone marrow, H: liver), locality, geographic coordinates, and number of sampled individuals (n) are indicated

\begin{tabular}{|c|c|c|c|c|c|}
\hline Taxa & $\begin{array}{l}\text { Tipo de } \\
\text { muestra }\end{array}$ & Localidad & País & $\begin{array}{l}\text { Ubicación } \\
\text { geográfica }\end{array}$ & $\begin{array}{l}\mathrm{n} \text { (total } \\
=423 \text { ) }\end{array}$ \\
\hline \multirow[t]{5}{*}{ L. g. cacsilensis } & $\mathrm{F}$ & Reserva Nacional de Calipuy, Departamento de La Libertad & Perú & $78^{\circ} 16^{\prime} \mathrm{O}, 08^{\circ} 27^{\prime} \mathrm{S}$ & 2 \\
\hline & $\mathrm{F}$ & Chavin, Departamento de Ica & Perú & $75^{\circ} 22^{\prime} \mathrm{O}, 14^{\circ} 39^{\prime} \mathrm{S}$ & 3 \\
\hline & $\mathrm{F}$ & Huallhua, Departamento de Ayacucho & Perú & $74^{\circ} 24^{\prime} \mathrm{O}, 14^{\circ} 39^{\prime} \mathrm{S}$ & 10 \\
\hline & $\mathrm{F}$ & Yarabamba, Arequipa, & Perú & $71^{\circ} 24^{\prime} \mathrm{O}, 16^{\circ} 04^{\prime} \mathrm{S}$ & 6 \\
\hline & $\mathrm{S}, \mathrm{F}$ & Putre, Precordillera de Arica, I Región & Chile & $69^{\circ} 35^{\prime} \mathrm{O}, 18^{\circ} 20^{\prime} \mathrm{S}$ & 20 \\
\hline \multirow[t]{9}{*}{ L. g. huanacus } & S & Paposo, costa al norte de Tal Tal, II Región & Chile & $70^{\circ} 30^{\prime} \mathrm{O}, 25^{\circ} 10^{\prime} \mathrm{S}$ & 1 \\
\hline & $\mathrm{F}$ & Parque Nacional Pan de Azúcar, III Región & Chile & $70^{\circ} 40^{\prime} \mathrm{O}, 26^{\circ} 07^{\prime} \mathrm{S}$ & 9 \\
\hline & $S, F, M$ & Parque Nacional Llanos de Challe, III Región & Chile & $71^{\circ} 05^{\prime} \mathrm{O}, 28^{\circ} 10^{\prime} \mathrm{S}$ & 6 \\
\hline & S & Vallenar, Comuna de Alto del Carmen, III Región & Chile & $70^{\circ} 56^{\prime} \mathrm{O}, 28^{\circ} 50^{\prime} \mathrm{S}$ & 4 \\
\hline & $\mathrm{F}$ & Llanos entre Ruta 5 y Puerto Choros, IV Región & Chile & $71^{\circ} 05^{\prime} \mathrm{O}, 29^{\circ} 25^{\prime} \mathrm{S}$ & 4 \\
\hline & $\mathrm{S}$ & Ovalle, IV Región & Chile & $70^{\circ} 30^{\prime} \mathrm{O}, 30^{\circ} 30^{\prime} \mathrm{S}$ & 4 \\
\hline & S & Minera Los Pelambres, Cordillera de Illapel, IV Región & Chile & $70^{\circ} 51^{\prime} \mathrm{O}, 31^{\circ} 71^{\prime} \mathrm{S}$ & 5 \\
\hline & S & Putaendo, Cordillera de San Felipe, V Región & Chile & $70^{\circ} 25^{\prime} \mathrm{O}, 32^{\circ} 30^{\prime} \mathrm{S}$ & 5 \\
\hline & $\mathrm{F}, \mathrm{MO}$ & Reserva Nacional Río Cipreses, VI Región & Chile & $70^{\circ} 20^{\prime} \mathrm{O}, 34^{\circ} 30^{\prime} \mathrm{S}$ & 6 \\
\hline \multirow[t]{8}{*}{ L. g. guanicoe } & $\mathrm{S}$ & Uspallata, Provincia de Mendoza & Argentina & $69^{\circ} 13^{\prime} \mathrm{O}, 32^{\circ} 50^{\prime} \mathrm{S}$ & 5 \\
\hline & S & Reserva Provincial La Payunia, Provincia de Mendoza & Argentina & $68^{\circ} 50^{\prime} \mathrm{O}, 36^{\circ} 10^{\prime} \mathrm{S}$ & 10 \\
\hline & $\mathrm{H}$ & Valle Chacabuco, XI Región & Chile & $72^{\circ} 27^{\prime} \mathrm{O}, 47^{\circ} 36^{\prime} \mathrm{S}$ & 19 \\
\hline & $S, M$ & Trelew, sector Bajada del Diablo, Provincia de Chubut & Argentina & $67^{\circ} 27^{\prime} \mathrm{O}, 42^{\circ} 49^{\prime} \mathrm{S}$ & 4 \\
\hline & S & Bariloche, Provincia de Neuquén & Argentina & $71^{\circ} 19^{\prime} \mathrm{O}, 41^{\circ} 09^{\prime} \mathrm{S}$ & 4 \\
\hline & $\mathrm{S}$ & Parque Nacional Torres del Paine, XII Región & Chile & $72^{\circ} 55^{\prime} \mathrm{O}, 51^{\circ} 03^{\prime} \mathrm{S}$ & 19 \\
\hline & $S, M$ & Tierra del Fuego, XII Región & Chile & $70^{\circ} 11^{\prime} \mathrm{O}, 53^{\circ} 18^{\prime} \mathrm{S}$ & 16 \\
\hline & $\mathrm{F}, \mathrm{M}$ & Isla Navarino, XII Región & Chile & $55^{\circ} 05^{\prime} \mathrm{O}, 67^{\circ} 15^{\prime} \mathrm{S}$ & 4 \\
\hline L. g. voglii & $\mathrm{F}$ & P. Nac. Kaa Iya, sureste del Chaco Boliviano & Bolivia & $62^{\circ} 26^{\prime} \mathrm{O}, 20^{\circ} 15^{\prime} \mathrm{S}$ & 6 \\
\hline \multirow[t]{14}{*}{ V.v.mensalis } & $\mathrm{S}$ & Catac, Departamento de Ancash & Perú & $77^{\circ} 17^{\prime} \mathrm{O}, 09^{\circ} 08^{\prime} \mathrm{S}$ & 8 \\
\hline & S & Sta. Ana de Tusi, Departamento de Cerro de Pasco & Perú & $76^{\circ} 21^{\prime} \mathrm{O}, 10^{\circ} 28^{\prime} \mathrm{S}$ & 15 \\
\hline & S & Huacarpana, Departamento de Ica & Perú & $75^{\circ} 04^{\prime} \mathrm{O}, 12^{\circ} 50^{\prime} \mathrm{S}$ & 17 \\
\hline & S & Tinco Cancha, Yauli, Departamento de Junín & Perú & $75^{\circ} 38^{\prime} \mathrm{O}, 11^{\circ} 02^{\prime} \mathrm{S}$ & 18 \\
\hline & S & Tingo Paccha, Jauja, Departamento de Junín & Perú & $75^{\circ} 27^{\prime} \mathrm{O}, 11^{\circ} 25^{\prime} \mathrm{S}$ & 18 \\
\hline & S & Parque Nacional Pampa Galeras, Departamento de Ayacucho & Perú & $75^{\circ} 24^{\prime} \mathrm{O}, 14^{\circ} 39^{\prime} \mathrm{S}$ & 20 \\
\hline & S & Huaytara, Departamento de Huancavelica & Perú & $75^{\circ} 15^{\prime} \mathrm{O}, 13^{\circ} 42^{\prime} \mathrm{S}$ & 8 \\
\hline & S & Puccro, Departamento de Cusco & Perú & $70^{\circ} 57^{\prime} \mathrm{O}, 14^{\circ} 10^{\prime} \mathrm{S}$ & 10 \\
\hline & S & Huacullani, Chucuito, Departamento de Puno & Perú & $69^{\circ} 20^{\prime} \mathrm{O}, 16^{\circ} 40^{\prime} \mathrm{S}$ & 8 \\
\hline & S & Aguada Blanca, Toccra, Departamento de Arequipa & Perú & $71^{\circ} 20^{\prime} \mathrm{O}, 16^{\circ} 10^{\prime} \mathrm{S}$ & 10 \\
\hline & S & Parque Nacional Lauca, I Región & Chile & $69^{\circ} 27^{\prime} \mathrm{O}, 18^{\circ} 10^{\prime} \mathrm{S}$ & 6 \\
\hline & S & Salar de Surire, I Región & Chile & $69^{\circ} 04^{\prime} \mathrm{O}, 18^{\circ} 55^{\prime} \mathrm{S}$ & 9 \\
\hline & S & Caquena, Comuna de Putre, I Región & Chile & $74^{\circ} 24^{\prime} \mathrm{O}, 14^{\circ} 39^{\prime} \mathrm{S}$ & 4 \\
\hline & S & Ankara, Comuna de General Lagos, I Región & Chile & $74^{\circ} 24^{\prime} \mathrm{O}, 14^{\circ} 39^{\prime} \mathrm{S}$ & 6 \\
\hline \multirow[t]{9}{*}{ V.v. vicugna } & S & Ollague, sector de Chela, II Región & Chile & $68^{\circ} 20^{\prime} \mathrm{O}, 21^{\circ} 20^{\prime} \mathrm{S}$ & 1 \\
\hline & S & Calama, II Región & Chile & $68^{\circ} 55^{\prime} \mathrm{O}, 22^{\circ} 26^{\prime} \mathrm{S}$ & 1 \\
\hline & S & Paso Jama, II Región & Chile & $67^{\circ} 07^{\prime} \mathrm{O}, 23^{\circ} 10^{\prime} \mathrm{S}$ & 1 \\
\hline & $\mathrm{S}$ & Parque Nacional Llullaillaco, II Región & Chile & $68^{\circ} 38^{\prime} \mathrm{O}, 24^{\circ} 28^{\prime} \mathrm{S}$ & 11 \\
\hline & $\mathrm{F}, \mathrm{M}$ & Parque Nacional Nevado Tres Cruces, III Región & Chile & $69^{\circ} 15^{\prime} \mathrm{O}, 27^{\circ} 53^{\prime} \mathrm{S}$ & 3 \\
\hline & S & INTA Abrapampa, Provincia de Jujuy & Argentina & $65^{\circ} 35^{\prime} \mathrm{O}, 22^{\circ} 20^{\prime} \mathrm{S}$ & 4 \\
\hline & S & Cieneguillas, Provincia de Jujuy & Argentina & $65^{\circ} 50^{\prime} \mathrm{O}, 22^{\circ} 06^{\prime} \mathrm{S}$ & 6 \\
\hline & S & Laguna Blanca, Provincia de Catamarca & Argentina & $66^{\circ} 05^{\prime} \mathrm{O}, 26^{\circ} 65^{\prime} \mathrm{S}$ & 3 \\
\hline & S & Reserva Provincial San Guillermo, Provincia de San Juan & Argentina & $67^{\circ} 35^{\prime} \mathrm{O}, 28^{\circ} 40^{\prime} \mathrm{S}$ & 2 \\
\hline \multirow[t]{2}{*}{ Lama glama } & S & & Chile & & 18 \\
\hline & S & & Perú & & 12 \\
\hline \multirow[t]{2}{*}{ Lama pacos } & S & & Chile & & 18 \\
\hline & $\mathrm{S}$ & & Perú & & 14 \\
\hline
\end{tabular}


Fragmentos de alrededor de 550 y 1.200 pb, correspondientes a la región control y citocromo $b$ del ADNmt, respectivamente, fueron amplificados mediante "la reacción en cadena de la polimerasa" (PCR, Saiki et al. 1985) usando protocolos "ad hoc" y partidores complementarios a la secuencia de ARNt y dominios conservados del ADNmt (Tabla 2, Marín 2004). Los fragmentos amplificados fueron purificados utilizando QIAGEN QIAquick, PCR Purification. La secuenciación de los productos de PCR se realizó en ambos sentidos, usando servicios de secuenciación automática, y utilizando como cebadores los mismos partidores utilizados para amplificar el fragmento.

Las secuencias obtenidas fueron alineadas utilizando el programa CLUSTAL-X (Thompson et al. 1997), SEQUENCHER (GeneCodes Corporation) y revisadas manualmente. El porcentaje de nucleótidos totales y por posición del codón, el número de transiciones (ts), el número de transversiones (tv) y las razones de estas (ts/tv) se calcularon con los programas MEGA-2 (Kumar et al. 2001) y PAUP 4.b8 (Swofford 1998). Los diferentes haplotipos fueron determinados usando el programa DnaSP 4.0 (Rozas et al. 2003). Las frecuencias haplotípicas, la diversidad haplotípica (h), el número de sitios polimórficos (S) y el número promedio de diferencia entre pares de secuencias (P) fueron calculados usando el programa ARLEQUIN (Schneider et al. 2000).

El análisis de las relaciones filogenéticas fue realizado con criterios de máxima parsimonia, máxima verosimilitud y distancia incluidos en el programa PAUP 4.b8, y por inferencia Bayesiana a través del programa MR. BAYES 2.01 (Huelsenbeck \& Ronquist 2001). Para la polarización de caracteres se utilizaron como grupos externos ("outgroups") las secuencias de Camelus dromedarius (X56281; Irwin et al. 1991) y Camelus bactrianus (U06427, Stanley et al. 1994) disponibles en GenBank.

El mejor modelo de evolución para las secuencias del gen citocromo $b$ obtenidas, fue determinado a través de una búsqueda anidada de entre 56 modelos incluidos en el programa MODELTEST 3.06 (Posada \& Crandall 1998). A partir de los análisis de verosimilitud obtenidos para cada modelo, se estableció que el modelo que mejor se ajustaba a las secuencias obtenidas correspondía a HKY85 + G. Considerando este modelo, fueron realizados todos los análisis filogenéticos del grupo y determinada la confiabilidad de los nodos en 500 réplicas de "bootstrap" (Felsenstein 1985). La probabilidad de cada nodo fue también calculada a través del muestreo entre un millón de generaciones con MR. BAYES 2.01.

TABLA 2

Secuencias de oligonucleótidos usadas como partidor para la amplificación del gen para citocromo $b(*)$ y el Dominio Hipervariable I de la Región Control (\#). Los nombres identifican el estándar de DNA para la cadena pesada (H) y liviana (L)

Oligonucleotide sequences used as primers for the amplification of cytochrome $b$ gene $(*)$ and Hypervariable Domine I of Control Region $(\#)$. L and $\mathrm{H}$ letters refer to the sequence of light and heavy strands, respectively

\begin{tabular}{|c|c|c|c|}
\hline $\begin{array}{l}\text { Número del } \\
\text { partidor }\end{array}$ & Nombre del partidor & Secuencia nucleotídica & $\begin{array}{l}\text { Temperatura de } \\
\text { alineamiento }\end{array}$ \\
\hline 1 & Lglu-ARTIO* & 5' TCTAACCACGACTAATGACATG $^{3}{ }^{\prime}$ & $68^{\circ} \mathrm{C}$ \\
\hline 2 & HThr-ARTIO* & 5' TCCTTTTTCGGCTTACAAGACC ${ }^{3}$ ' & $69^{\circ} \mathrm{C}$ \\
\hline 3 & L $400 *$ & 5' GGGCTATGTACTCCCATGAGG ${ }^{3}{ }^{\prime}$ & $73^{\circ} \mathrm{C}$ \\
\hline 4 & LBE- $02 *$ & 5' CTCCGTAGATAAAGCCACCC ${ }^{3}{ }^{\prime}$ & $64{ }^{\circ} \mathrm{C}$ \\
\hline 5 & $\mathrm{H} 600 *$ & 5' TTGTTGGAGCCTGTTTCGTG ${ }^{3}$ & $84{ }^{\circ} \mathrm{C}$ \\
\hline 6 & L1140G* & 5' CCCTAATCCTAATTTTAATGCC ${ }^{3}$ & $66^{\circ} \mathrm{C}$ \\
\hline 7 & LThr-ARTIO $\#$ & 5' GGTCTTGTAAGCCGAAAAAGGA ${ }^{3}$ ' & $65^{\circ} \mathrm{C}$ \\
\hline 8 & Lloop0007G ${ }^{\#}$ & 5' GTACTAAAAGAAAATATCATGTC ${ }^{3}$ ' & $63{ }^{\circ} \mathrm{C}$ \\
\hline 9 & Lloop0007V"\# & 5' GTACTAAAAGAGAATTCTATGTC ${ }^{3}$ ' & $65^{\circ} \mathrm{C}$ \\
\hline 10 & Lloop310-ARTIO $\#$ & 5' TAGATCACGAGCTTAATCACC ${ }^{3}$ & $67{ }^{\circ} \mathrm{C}$ \\
\hline 11 & Hloop550G $\#$ & $5^{\prime}$ ATGGACTGAATAGCACCTTATG ${ }^{3}$ ' & $78^{\circ} \mathrm{C}$ \\
\hline 12 & $\mathrm{H} 362^{\#}$ & 5' GGTTTCACGCGGCATGGTGATT ${ }^{3}$ ' & $73^{\circ} \mathrm{C}$ \\
\hline
\end{tabular}


Una genealogía en red de haplotipos del gen para el Dominio Hipervariable I de la Región Control fue inferida usando el algoritmo "median joining network" implementada en el programa NETWORK, versión 2.0 (Bandelt et al. 1999). Con este programa todos los árboles de expansión mínima son combinados dentro de una red, siguiendo un algoritmo análogo al propuesto por Excoffier \& Smouse (1994).

\section{RESULTADOS}

\section{Cromosomas bandeados $G$}

El análisis cromosómico de las cuatro especies de camélidos sudamericanos indicó un $2 \mathrm{n}=74$. Los 20 primeros pares y el cromosoma $\mathrm{Y}$ de los machos presentaban centrómeros en posición subterminal o terminal; los restantes 16 pares y el cromosoma $\mathrm{X}$ presentaron centrómeros en posición submedial o medial. En general, las bandas $\mathrm{G}$ presentaron patrones similares en las cuatro especies (Fig. 1), incluso similares a las descritas para el camello, Camelus bactrianus. Sin embargo, fue posible observar finas y consistentes diferencias en el patrón de bandas $\mathrm{G}$ de los brazos cortos del par 1 de guanaco y vicuña. En efecto, en el primero se observó una banda $\mathrm{G}$ oscura terminal en los dos homólogos de todos los individuos, denominada aquí 1p13 (Fig. 2 ); en contraste, todas las vicuñas presentaban una banda clara terminal en esta posición, y una banda adicional oscura $1 \mathrm{p} 12$ cercana al centrómero (Fig. 1 y 2). Ese patrón en guanaco también fue observado consistentemente en todas las llamas aquí analizadas, en la célula de camello incluida para comparación (Fig. 1), así como en uno de los homólogos del híbrido guanaco $\mathrm{x}$ alpaca (Fig. 2). El patrón de vicuña fue observado también en todas las alpacas analizadas aquí (Fig. 2 ), así como en el otro homólogo del par 1 en el híbrido guanaco x alpaca (Fig. 2).

\section{Secuencias del gen para citocromo $b$}

El promedio de la relación ts/tv (transiciones/ transversiones) observada entre todos los taxa fue de 8,85; es decir, que por cada transversión existen casi nueve transiciones. Considerando solo los camélidos del Nuevo Mundo, el número de transiciones promedio fue de 39,66, el número de transversiones promedio fue de
4,35 y la relación ts/tv de 9,27; es decir, que por cada transversión existen nueve transiciones. El valor mínimo de esta razón $(3,53)$ estuvo entre las vicuñas, donde el número de transiciones y transversiones promedio fue de 14,60 y 4,47 respectivamente. Los guanacos, por su parte, mostraron 10,04 transiciones promedio y 1,50 transversiones promedio, con una relación ts/tv de 7,18.

Considerando la secuencia completa del gen para citocromo $b$ en todos los taxa, la composición de bases entre ellos fue similar, como evidencia el test de chi-cuadrado de homogeneidad $\left(\chi^{2}=8,953427 ; \mathrm{gl}=156 ; \mathrm{P}=\right.$ $1,00)$. Al analizar las secuencias, tomando en cuenta la posición de cada nucleótido en el triplete y los distintos taxa, la composición promedio de nucleótidos para la hebra liviana, incluyendo a los grupos externos, fue de $28,5 \%$ de adenina, 29,7\% de timina, 27,8\% de citosina y $14,1 \%$ de guanina, constatándose un significativo déficit de guaninas $(\mathrm{P}<0,001)$ usando la prueba de $\chi^{2}$. Esta observación se acentuó en la tercera posición del triplete $\quad(4,8$ $\%)$ de todos los taxa, concordando con lo observado en otros mamíferos (Irwin et al. 1991).

La topología del árbol obtenido con el algoritmo de unión del vecino más próximo ("Neighbor-Joining"), usando el modelo de evolución nucleotídica de Hasegawa-KishinoYano (HKY85+G) (Hasegawa et al. 1985), y considerando las tres posiciones de codón, recogió a $V$. vicugna como taxón hermano de L. guanicoe, reconociéndose a la tribu Camelini como grupo externo de ambos; siendo L. guanicoe y $V$. vicugna recíprocamente monofiléticos. Por su parte, todos los análisis recogieron a L. glama (llama) como taxón hermano de L. guanicoe y a $V$. pacos (alpaca) como parte del mismo clado formado por las vicuñas mensalis. El análisis de las secuencias del gen para citocromo $b$ mostró dos clados entre las poblaciones de $V$. vicugna, los que concuerdan con las dos subespecies previamente reconocidas para esta especie.

Sin embargo, los resultados obtenidos del análisis de poblaciones de L. guanicoe no mostraron una separación subespecífica concordante con las cuatro subespecies definidas para la especie (Fig. 3). Con el criterio de parsimonia máxima y usando la aproximación "branch-and-bound", se obtuvieron nueve árboles igualmente parsimoniosos de 406 pasos cada uno, de entre 447 árboles evaluados, con un 


\section{G L V A}

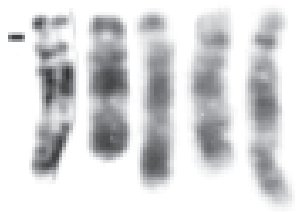

1

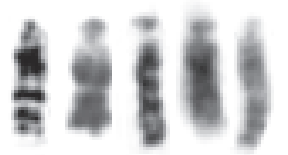

5

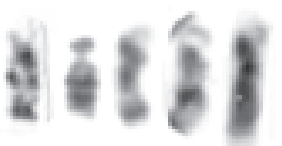

9

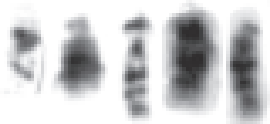

13

है, और

17

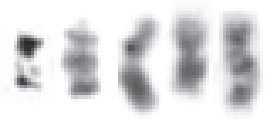

21

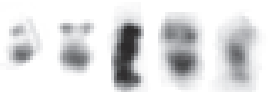

25

$-5:$ i 4

29

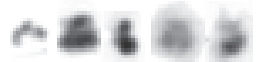

33

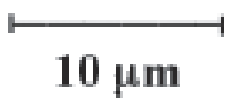

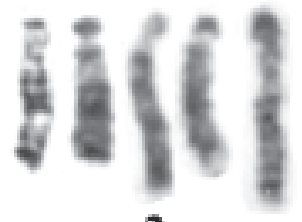

2

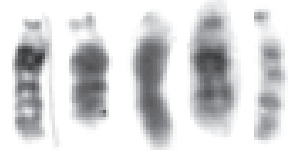

6

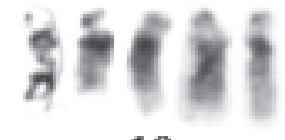

10

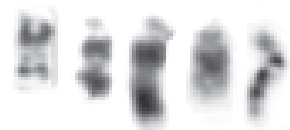

14

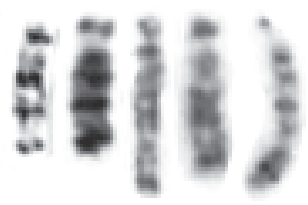

3

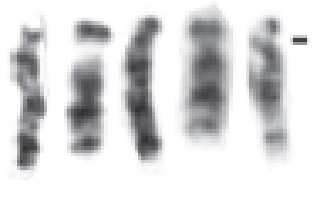

4

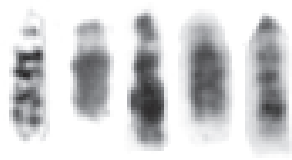

7

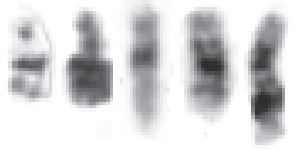

8
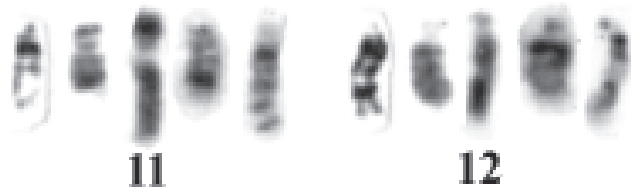

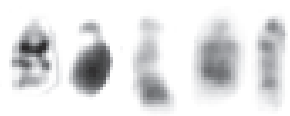

15
E2 2 ह

16
* is 06

18

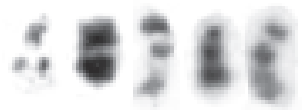

22

1818 :

26

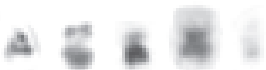

30

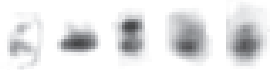

34 ća

19

20

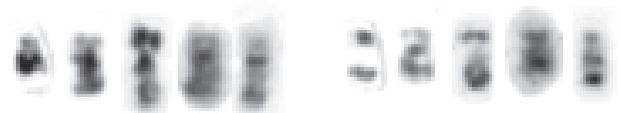

23

24

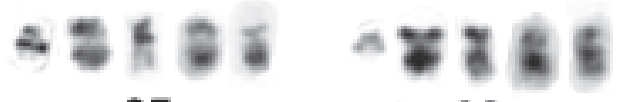

27

28

$-3 \mathrm{E}=\mathrm{y}$

31

4. 53 is

32

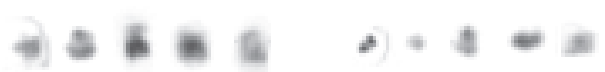

35

36

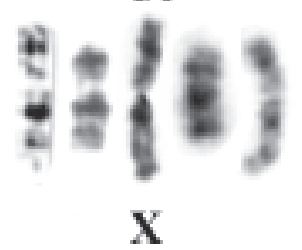

Fig. 1: Cariotipos haploides con bandas G comparados; C: camello; G: guanaco; L: llama; V: vicuña; A: alpaca.

Haploid karyotypes with G-bands; C: camel; G: guanaco; L: llama; V: vicuña; A: alpaca. 


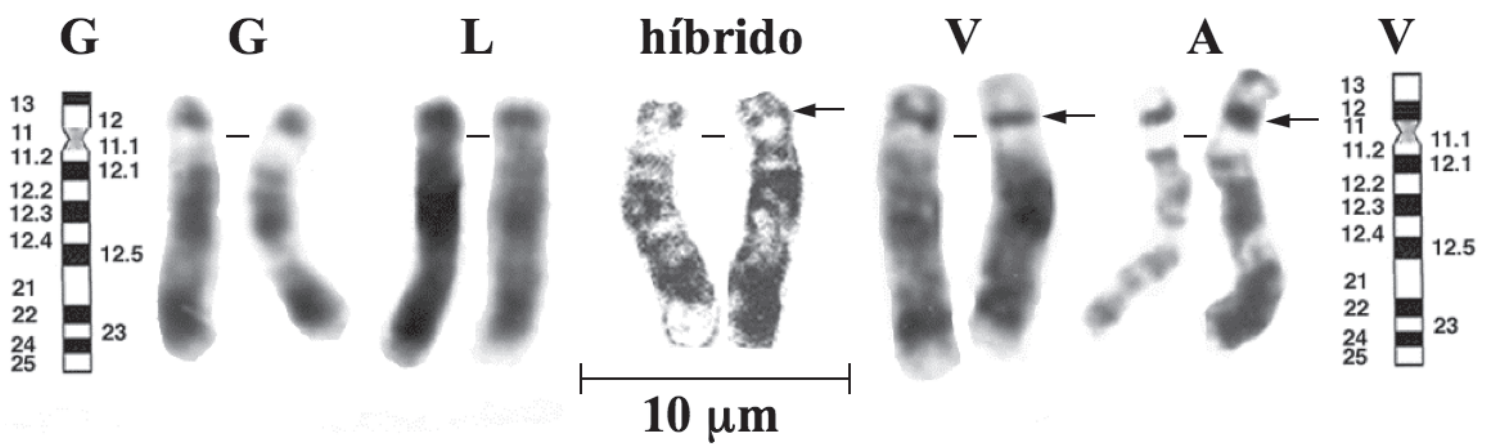

Fig. 2: Bandas $\mathrm{G}$ en el par cromosómico 1 de camélidos sudamericanos. Al centro par 1 de híbrido guanaco x alpaca; la flecha marca la banda proximal oscura 1p12, presente en alpaca y vicuña. Al extremo derecho del ideograma bandeado de vicuña; al extremo izquierdo del idiograma bandeado de guanaco.

G-bands in chromosome pair 1 from South American camelids. Center, pair 1 of guanaco x alpaca hybrid, arrowhead points proximal dark band $1 \mathrm{p} 12$, observed in alpaca and vicuña. Extreme right, banded ideogram of vicuña; extreme left, banded ideogram of guanaco.

Índice de Consistencia $(\mathrm{CI})=0,704$, un Índice de Homoplasia $(\mathrm{HI})=0,296$ y un Índice de Retención $(\mathrm{RI})=0,612$, con un valor de evolución mínima de 0,971. Todos los árboles mostraron una topología similar a la obtenida por análisis de distancias. Igualmente, el análisis Bayesiano no mostró diferencias sustanciales, reafirmando lo encontrado por Kadwell et al. (2001) donde $V$. vicugna y L. guanicoe se presentan como grupos monofiléticos, con un 87 y $100 \%$ de probabilidad, respectivamente. En discordancia con estos análisis, el uso del programa PAUP para la búsqueda del árbol más verosímil, considerando todos los caracteres, recogió a vicuñas como polifiléticas (árbol no mostrado); sin embargo, la sustentación de estos nodos fue inferior al $50 \%$ en 100 réplicas de "bootstrap".

\section{Secuencias del Dominio Hipervariable I de la Región Control}

Considerando un fragmento de $328 \mathrm{pb}$ e incluyendo la inserción de una timina detectada en algunos guanacos y llamas, 77 haplotipos distintos fueron revelados entre las secuencias del Dominio Hipervariable I de 423 especímenes silvestres y domésticos (Tabla 3). De ellos, 34 haplotipos fueron detectados en vicuñas ( $\mathrm{n}=$ 189); 34 en guanacos $(\mathrm{n}=172)$; seis en llamas $(\mathrm{n}=30)$ y ocho en alpacas $(\mathrm{n}=32)$; de ellos, seis se mostraron compartidos entre $V$. vicugna mensalis y alpacas, dos compartidos entre llamas y alpacas y uno compartido entre guanacos y llamas (Tabla 3).
El diagrama del árbol de expansión mínima de los haplotipos ("network") reveló dos grandes grupos de haplotipos separados por 10 pasos mutacionales (Fig. 4). Uno de ellos reúne el conjunto de los haplotipos revelados en guanacos en unión con la totalidad de los haplotipos detectados en llamas (Haplogrupo 1). Dos de estos haplotipos fueron detectados en un gran número de ejemplares $(\mathrm{n}=43$ y $\mathrm{n}=$ 29) de un amplio rango de distribución, incluyendo en ellos representantes de L. $g$. voglii, L. g. huanacus y L. g. guanicoe. De los 39 haplotipos hallados, solo uno de ellos fue compartido por ocho guanacos y 3 llamas, mostrando ausencia de estructuración entre ellos. Este haplogrupo mostró también haplotipos compartidos entre llamas e individuos fenotípicamente reconocidos como alpaca.

El segundo grupo reúne a vicuñas y alpacas (Haplogrupo 2). A diferencia del haplogrupo formado entre guanacos y llamas, se observó, al interior de este haplogrupo, la presencia de dos cluster formados principalmente por $V$. $v$. mensalis y $V . v$. vicugna, respectivamente. No se observaron haplotipos compartidos entre alpacas y llamas en este haplogrupo; sin embargo, se observó la presencia de seis haplotipos compartidos entre vicuñas mensalis y alpacas. Sin excepción, todos los haplotipos detectados en alpacas forman parte del cluster formado por las vicuñas más septentrionales. Aquí se muestra también un haplotipo dominante representado en 130 individuos, algunos de los cuales correspondieron al fenotipo alpaca. 
TABLA 3

Variación de 56 sitios polimórficos entre 77 haplotipos obtenidos a partir de la secuencia del Dominio Hipervariable I (Región Control, mtDNA) de camélidos sudamericanos, mostrando solo los sitios variables. Por cada haplotipo se indican el número de individuos que presentan cada forma haplotípica y la especie a la cual pertenecen. v: Vicugna vicugna (vicuña); a: Lama pacos; g: Lama guanicoe; 1: Lama glama

Variation at 56 polymorphic sites among 77 haplotypes of South American camelids. Hypervariable I Domain sequences (Control Region, mtDNA). For each haplotype, the total number of individuals with an haplotype is indicated, and its species: v, Vicugna vicugna (vicuña); h, Vicugna pacos (alpaca); g, Lama guanicoe (guanaco); 1, Lama glama (1lama)

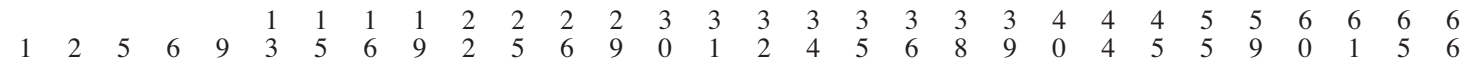

H1

H2

$\mathrm{H} 3$

$\mathrm{H} 4$

H5

H6

H7

$\mathrm{H} 8$

H9

H10

H1 1

H12

H13

H14

H15

H16

H17

H18

H19

H20

H21

H 22

H 23

$\mathrm{H} 24$

H 25

H26

H27

H28

H29

H30

H31

H32

H33

H34

H35

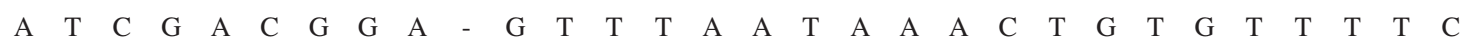
$\mathrm{A}$

A

A

G

A G

C C

$\mathrm{C} \cdot \mathrm{C}$

C

C

C

C C

A $\mathrm{G}$

C C

A $G$

C C

A $\mathrm{G}$

A

G 
$\begin{array}{llllllllllllllllllllllllll}6 & 6 & 7 & 7 & 8 & 8 & 9 & 1 & 1 & 1 & 1 & 1 & 1 & 2 & 2 & 2 & 2 & 2 & 2 & 2 & 2 & 2 & 2 & 2 & 2 & 3 \\ 8 & 9 & 0 & 3 & 2 & 5 & 1 & 6 & 5 & 2 & 1 & 2 & 6 & 1 & 1 & 1 & 3 & 3 & 3 & 4 & 5 & 6 & 7 & 8 & 9 & 2 \\ \end{array}$

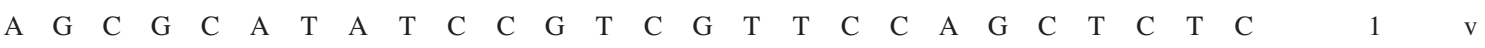

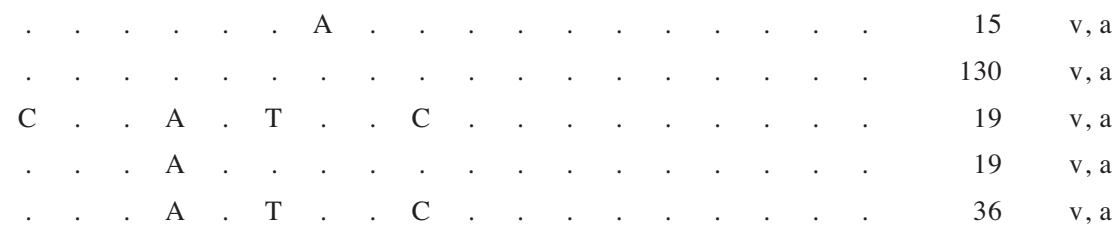

G A T

$\mathrm{A} . \mathrm{C}$

A $\mathrm{T}$

A C $\mathrm{T}$

A $\mathrm{T}$

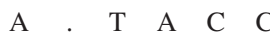

A $\mathrm{T}$

$\mathrm{A} \cdot \mathrm{A} \quad \mathrm{C}$

A $\mathrm{T}$

A . T A C

A $\quad$ C $T$ A

. $\mathrm{A} \mathrm{T}$

$\mathrm{C}$

A $\mathrm{C}$ T A

G A $\mathrm{T}$

$\mathrm{C}$

A $\mathrm{C}$ T

A

A . T

C

A

A C

C. $. \mathrm{A} . \mathrm{T} . \mathrm{C}$

A . T

.

. $\mathrm{A}$ T $. \mathrm{G} . \quad \mathrm{C} . \quad \mathrm{A} . \quad \mathrm{A} C \mathrm{C}$

A $\mathrm{T}$

A $\mathrm{C}$ T A

G A $\mathrm{T}$

A $\mathrm{C} T$

$\mathrm{C} . \mathrm{A} \quad \mathrm{C} \mathrm{T}$

G A T

A $\mathrm{T}$

A . T A C C

G A T

$\mathrm{A} \cdot \mathrm{T}$

A $\mathrm{T}$

A

A C

G A T

A . T

C

$\mathrm{A} . \mathrm{A} . \mathrm{A}$ 


\begin{tabular}{|c|c|c|c|c|c|c|c|c|c|c|c|c|c|c|c|c|c|c|c|c|c|c|c|c|c|c|c|c|}
\hline & 1 & 2 & 5 & 6 & 9 & $\begin{array}{l}1 \\
3\end{array}$ & $\begin{array}{ll}1 & 1 \\
5 & 6\end{array}$ & $\begin{array}{l}1 \\
9\end{array}$ & $\begin{array}{l}2 \\
2\end{array}$ & $\begin{array}{l}2 \\
5\end{array}$ & $\begin{array}{l}2 \\
6\end{array}$ & $\begin{array}{l}2 \\
9\end{array}$ & $\begin{array}{l}3 \\
0\end{array}$ & $\begin{array}{l}3 \\
1\end{array}$ & $\begin{array}{l}3 \\
2\end{array}$ & $\begin{array}{l}3 \\
4\end{array}$ & $\begin{array}{l}3 \\
5\end{array}$ & $\begin{array}{l}3 \\
6\end{array}$ & $\begin{array}{l}3 \\
8\end{array}$ & $\begin{array}{l}3 \\
9\end{array}$ & $\begin{array}{l}4 \\
0\end{array}$ & & $\begin{array}{l}4 \\
5\end{array}$ & $\begin{array}{l}5 \\
5\end{array}$ & $\begin{array}{l}5 \\
9\end{array}$ & $\begin{array}{l}6 \\
0\end{array}$ & $\begin{array}{l}6 \\
1\end{array}$ & $\begin{array}{l}6 \\
5\end{array}$ \\
\hline H36 & . & . & . & A & . & & . & G & - & & C & . & C & G & . & C & . & & G & & C & & . & A & A & & C & . \\
\hline H37 & . & . & . & A & . & & . & G & - & & . & . & . & G & . & $\mathrm{C}$ & G & G & . & $\mathrm{T}$ & . & & . & A & A & . & C & . \\
\hline H38 & . & . & . & A & . & & . & G & - & . & . & . & . & G & . & . & . & & G & $\mathrm{T}$ & . & & . & A & A & . & $\mathrm{C}$ & \\
\hline H39 & & . & . & A & & . & . & G & - & & . & & & G & & . & & . & G & $\mathrm{T}$ & & & . & A & A & . & C & \\
\hline $\mathrm{H} 40$ & . & . & . & A & . & . & . & G & - & . & C & . & C & G & . & C & . & . & G & . & C & & . & A & A & . & C & \\
\hline $\mathrm{H} 41$ & . & . & . & A & . & & . & $\mathrm{G}$ & - & . & . & . & . & G & . & . & . & . & G & G & . & & . & A & A & . & $\mathrm{C}$ & \\
\hline $\mathrm{H} 42$ & . & . & . & A & . & & . & $\mathrm{G}$ & - & . & . & . & C & G & . & C & . & . & G & $\mathrm{T}$ & . & & . & A & A & & C & . \\
\hline $\mathrm{H} 43$ & . & . & . & A & . & . & . & G & - & . & . & . & . & G & . & C & . & . & G & . & . & & . & A & A & . & C & . \\
\hline $\mathrm{H} 44$ & . & . & . & A & . & . & . & $\mathrm{G}$ & - & . & . & . & . & G & . & $\mathrm{C}$ & . & . & . & $\mathrm{T}$ & . & & . & A & A & . & $\mathrm{C}$ & \\
\hline $\mathrm{H} 45$ & . & . & . & A & . & & . & $\mathrm{G}$ & - & . & . & . & . & G & . & . & . & . & G & $\mathrm{T}$ & . & & . & A & A & . & C & . \\
\hline $\mathrm{H} 46$ & . & . & . & A & . & & . & G & $\mathrm{T}$ & . & . & . & . & G & . & C & $\mathrm{G}$ & G & . & $\mathrm{T}$ & . & &.$\quad$ & A & A & . & C & . \\
\hline $\mathrm{H} 47$ & . & . & . & A & . & & . & G & - & . & . & . & . & G & . & C & . & . & G & & . & &.$\quad$ & A & A & & $\mathrm{C}$ & \\
\hline $\mathrm{H} 48$ & . & . & . & A & . & & . & G & - & . &. & . & . & G & . & C & . & . & G & & . & & A & A & A & . & C & . \\
\hline $\mathrm{H} 49$ & & . & . & A & & . & . & G & - & & . & & & G & & C & & . & G & . & & &.$\quad$ & A & A & . & C & \\
\hline H50 & . & . & . & A & . & & . & $\mathrm{G}$ & - & . & . & . & . & G & . & C & . & . & G & & . & 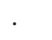 & A & A & A & . & C & \\
\hline H51 & . & . & . & A & . & & . & G & - & A & . & . & . & G & . & C & . & . & G & & . & $\mathrm{T}$ & A & A & A & . & C & . \\
\hline H52 & . & . & . & A & . & & . & $\mathrm{G}$ & - & . & . & . & . & G & . & $\mathrm{C}$ & . & . & G & & . & & . & A & A & . & C & . \\
\hline H53 & . & . & . & A & . & . & . & G & - & . & . & . & & G & & $\mathrm{C}$ & . & . & G & . & & & . & A & A & . & C & \\
\hline H54 & . & 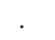 & . & A & . & . & . & G & - & & . & . & . & G & . & C & . & . & G & . & . & & . & A & A & . & C & \\
\hline H55 & . & . & . & A & . & & . & $\mathrm{G}$ & - & . & & . & . & G & . & $\mathrm{C}$ & . & . & G & & . & & . & A & A & & C & . \\
\hline H56 & . & . & G & A & . & & . & $\mathrm{G}$ & - & . & . & . & . & G & . & C & . & . & G & & . & & . & A & A & . & C & . \\
\hline H57 & . & . & . & A & . & . & . & G & - & . & . & . & . & G & . & C & . & . & G & . & . & & . & A & A & . & C & . \\
\hline H58 & . & . & . & A & . & . & . & $\mathrm{G}$ & - & . & . & . & . & $\mathrm{G}$ & . & C & . & . & G & & . & & . & A & A & . & C & . \\
\hline H59 & . & . & . & A & . & . & . & $\mathrm{G}$ & - & . & . & . & & $\mathrm{G}$ & . & C & . & . & G & . & & &.$\quad$ & A & A & . & C & $\mathrm{C}$ \\
\hline H60 & . & . & . & A & . & & . & G & - & & . & . & . & G & . & C & . & . & G & & . & & A & A & A & & C & . \\
\hline H61 & C & C & . & A & . & & . & $\mathrm{G}$ & - & . & . & . & . & G & . & C & . & . & G & & . & & A & A & A & . & C & . \\
\hline H62 & $\mathrm{T}$ & & . & A & . & & . & $\mathrm{G}$ & - & . & . & . & . & $\mathrm{G}$ & . & $\mathrm{C}$ & . & . & G & & . & &.$\quad$ & A & A & . & C & . \\
\hline H63 & & & & A & & . & A & G & - & & . & & & G & & C & & & G & . & & & A & A & A & . & C & \\
\hline H64 & . & & . & A & . & & . & G & - & & . & . & . & $\mathrm{G}$ & . & C & . & . & G & & . & & A & A & A & . & C & . \\
\hline H65 & . & . & . & A & . & . & . & $\mathrm{G}$ & - & 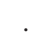 & . & . & . & G & . & $\mathrm{C}$ & . & . & G & . & . & & A & A & A & . & C & . \\
\hline H66 & . & C & . & A & . & & . & $\mathrm{G}$ & - & . & . & . & . & G & . & $\mathrm{C}$ & . & . & G & & . & & . & A & A & & C & . \\
\hline H67 & . & C & . & A & . & . & . & G & - & . & . & . & . & G & . & C & . & . & G & . & . & & . & A & A & . & C & . \\
\hline H68 & . & & . & A & . & . & . & $\mathrm{G}$ & - & & . & . & . & $\mathrm{G}$ & . & $\mathrm{C}$ & . & . & G & . & . & & A & A & A & . & C & . \\
\hline H69 & . & & . & A & . & . & . & $\mathrm{G}$ & - & & . & . & . & G & . & $\mathrm{C}$ & . & . & G & & . & & . & A & A & . & C & . \\
\hline H70 & . & $\cdot$ & . & A & . & . & . & G & - & ${ }^{\circ}$ & . & . & . & G & . & C & . & . & G & . & . & & . & A & A & . & C & . \\
\hline H71 & . & . & . & A & . & & . & G & - & & & . & . & G & . & . & . & . & G & $\mathrm{T}$ & . & & . & A & A & & C & \\
\hline H72 & . & & . & A & . & & . & G & - & & . & . & $\mathrm{C}$ & G & . & C & . & . & G & & . & & . & A & A & . & C & \\
\hline H73 & & . & . & A & & . & . & G & - & . & . & . & & G & & $\mathrm{C}$ & & . & & $\mathrm{T}$ & & & . & A & A & . & C & \\
\hline H74 & & & & A & & . & . & G & - & & . & . & & G & & C & & & G & . & & & . & A & A & . & C & \\
\hline H75 & . & & . & . & . & & A & . & - & & . & . & . & & . & . & . & & . & & . & & . & & . & . & & \\
\hline $\mathrm{H} 76$ & . & & . & . & . & & A & . & - & & . & . & . & & G & & . & & & & . & & . & & . & . & & \\
\hline H77 & & . & . & . & & . & A & . & - & . & . & . & & . & & & . & & & . & & & . & . & . & . & & \\
\hline
\end{tabular}




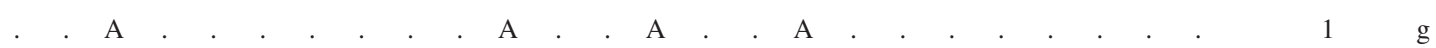

$\mathrm{A} . \mathrm{A} . \quad \mathrm{C} . \mathrm{A}$.

$\mathrm{A} \cdot \mathrm{A} \cdot \mathrm{A}$

$\mathrm{A} \cdot \mathrm{A} \cdot \mathrm{C}$

$\mathrm{A} \mathrm{C} \cdot \mathrm{A} \cdot \mathrm{A}$

$\mathrm{A} \cdot \mathrm{A} \cdot \mathrm{C}$

$\mathrm{A} \mathrm{C} \cdot \mathrm{A} \cdot \mathrm{A}$

$\mathrm{A} \cdot \mathrm{A} \cdot \mathrm{A}$

$\mathrm{A} \cdot \mathrm{A} \cdot \mathrm{C}$

$\mathrm{A} \mathrm{C} \cdot \mathrm{A}$.

A

$\mathrm{A} \cdot \mathrm{A} \cdot \mathrm{C}$

$\mathrm{A} \cdot \mathrm{A} \mathrm{C} \cdot \mathrm{A}$

$\mathrm{A} \cdot \mathrm{A} \cdot \cdot \cdot \cdot \cdot \cdot \mathrm{A} \cdot \mathrm{A} \cdot \mathrm{A} \cdot \mathrm{A}$

$\mathrm{A} \cdot \mathrm{A} \cdot \mathrm{C} \cdot \mathrm{C} \cdot \mathrm{A} \cdot \mathrm{A} \cdot \mathrm{A} \cdot \mathrm{A}$

$\mathrm{A} \cdot \mathrm{A} \cdot \mathrm{C} \cdot \mathrm{C}^{-} \cdot \mathrm{A} \cdot \mathrm{A} \cdot \mathrm{C} \cdot \mathrm{A}$

$\mathrm{A} \cdot \mathrm{A} \cdot \mathrm{C} \cdot \mathrm{A} \cdot \mathrm{A} \cdot \mathrm{A} \cdot \mathrm{A} \cdot \mathrm{A}$

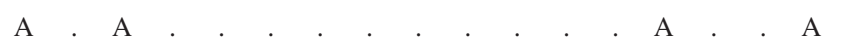

$\mathrm{A} . \mathrm{A} . . \quad . \quad . \quad . \quad . \quad . \mathrm{A} . \mathrm{A}$

$\mathrm{A} . \mathrm{A} \cdot . \quad . \quad . \quad . \mathrm{A} . \quad . \mathrm{A} . . \mathrm{A}$

A . A

A

$\mathrm{A} \cdot \mathrm{A}$

A . A

$\mathrm{A}$

$\mathrm{A} . \mathrm{A}$

A . A

$\mathrm{A} \cdot \mathrm{A}$

A

$\mathrm{A} \cdot \mathrm{A}$

$\mathrm{A} . \mathrm{A} \mathrm{T}$

$\mathrm{A} \cdot \mathrm{T} \mathrm{A} \cdot \mathrm{A}$

A . A

$\mathrm{A} \cdot \mathrm{A}$ T

$\mathrm{A} \cdot \mathrm{A} . \mathrm{G}$

$\mathrm{A} . \mathrm{A} . \mathrm{C}$.

$\mathrm{A} \cdot \mathrm{A}$

A . A

$\mathrm{A} \cdot \mathrm{A}$

A . A

$\mathrm{A} \cdot \mathrm{A}$

C

$\mathrm{A} \cdot \mathrm{A}$

A . A

$\mathrm{A} . \mathrm{A}$

$\mathrm{A} \cdot \mathrm{A}$

$\mathrm{A} \cdot \mathrm{A}$

A . A

$\mathrm{A} \cdot \mathrm{A}$

$A . A . G$

A . A . C

$\mathrm{A} \cdot \mathrm{A} \cdot \mathrm{C}$

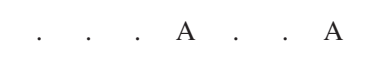

$\mathrm{A} . \mathrm{A}$

$\mathrm{A} \cdot \mathrm{A}$ T

$\cdot$. $\mathrm{A} \cdot \mathrm{A}$

$\mathrm{A} \cdot . \mathrm{A} \cdot \mathrm{A}$

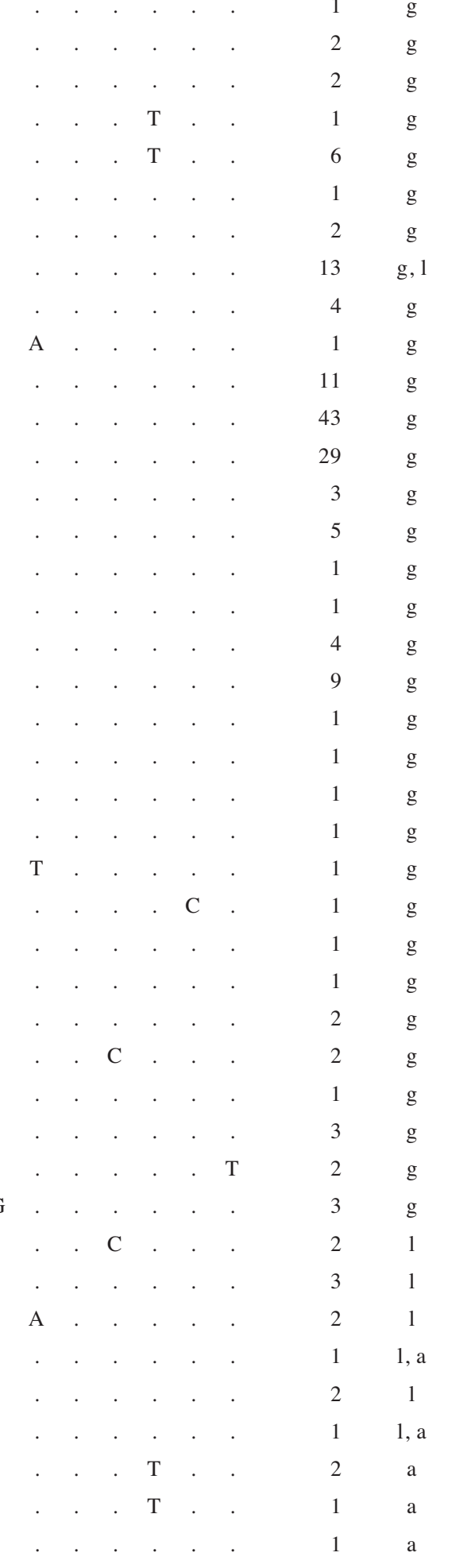

$\mathrm{A} \cdot \mathrm{A}$

$\mathrm{A} \cdot \cdot \cdot \mathrm{A} \cdot \cdot \cdot \mathrm{A}$

$\mathrm{A} \cdot \mathrm{A} \cdot \mathrm{A}$

$\mathrm{A} . \quad \mathrm{A} . \mathrm{A}$

$\mathrm{A} \cdot \mathrm{A}$

$\mathrm{A} . \mathrm{A}$

. A . $\mathrm{A}$

A . G

. A . A

$\mathrm{A} \cdot . \mathrm{A} \cdot \mathrm{A}$

$\mathrm{A} \cdot \cdot \mathrm{A} \cdot \mathrm{A}$

$\mathrm{A} \cdot \mathrm{A} \mathrm{C} \cdot \mathrm{A}$

$\mathrm{A} . \mathrm{T} \mathrm{A} . \mathrm{A}$

C

. 
NJ

L. g. cacsilencis

$\Delta$ L. g. huanacus

○ L. g. voglii

L.g. guanicoe

फ $V . v$. mensalis

\& $V$.v. vicugna \$unín

- $\$$ Puno

$\$$ Paquisa 2

- 4 Caquena 2

QPampa Galeras

$\$$ Caquena 1

$\$$ Paquisa 1

Lama pacos Suri

96/1.00 Lama pacos Huacaya

99/1.00 L Catamarca 3

- 1 Ica

72/0.89 $\$$ Huancavelica

\& Calama
\& Paso Jama

$100 / 0.87$

100/1.00
$\propto$ III Reg. Cord.

c San Juan 3

- \&Llullaillaco 2

C Cieneguillas 1

¿ $<$ Jujui

c Catamarca 2

Llullaillaco 10

100/1.00 San Juan 2

100/1.00 Huaiihua 05

- Huaiihua 11

97/1.00 Dutre 1

$\Delta$ Pan de Azúcar

63/0.84 $\bigcirc$ Kaa Iya 51

$\mathbf{\Delta}$ Huasco Cord. 1

$\Delta$ Vallenar 1

100/1.00

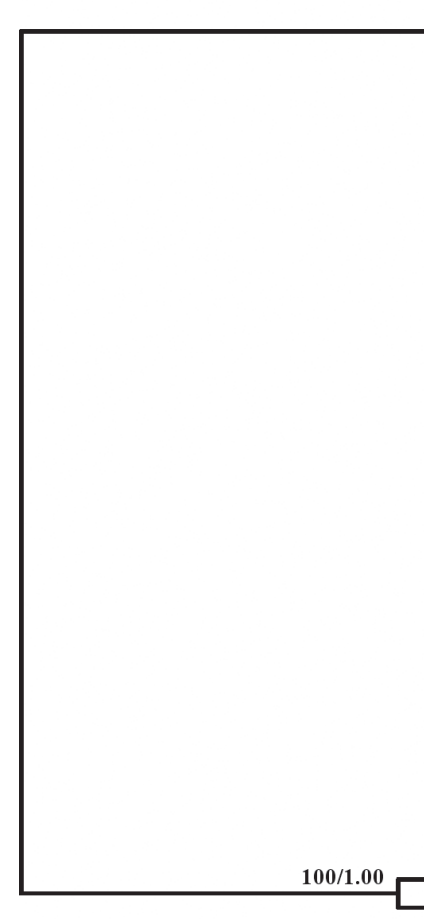

$1 / .5$

54/4.56- $\begin{aligned} & \text { O Trelew } \\ & \text { O Tierra del Fuego } 1\end{aligned}$

$72 / 0.89-151$ O Tierra del Fuego

$\Delta$ Huasco Costa

$\Delta$ Illapel Cord. 1

OValle Chacabuco 1

OUspallata

- Kaa Iya 08

$\Delta$ Llanos de Challe

$\Delta$ Huasco Cord. 2

$\Delta$ Huasco Costa 1

$\Delta$ Vallenar 2

OBariloche

OValle Chacabuco 2

- O PN Torres del Paine

100/1.00

100/1.00

8 / 10.93 - $\Delta$ Ovalle 2

$\Delta$ San Felipe Cord.

$\mathbf{b} \mathbf{\Delta}$ Illapel Cord. 2

92/0.96 Lama glama Chaku

- $\Delta$ Punta de Choros

Putre 2

$\Delta$ Paposo

69/0.73 $\$ 1 / 1.00 — L a m a$ glama Ccara

Lama glama Arg

Camelus bactrianus

Camelus dromedarius
Clado 1:

Vicuñas

$+$

Alpacas
Clado 2:

Guanacos $+$

Llamas

Fig. 3: Árbol de distancia (Neighbour-Joining) representando las relaciones filogenéticas de camélidos sudamericanos basados en las secuencias del gen citocromo $b$ (Modelo HKY+G). Los números en los nodos representan valores bootstrap ( $>50 \%, 1.000$ réplicas) y de probabilidad derivados de análisis Bayesiano. Los taxa son indicados a la derecha.

Neighbour-joining tree representing phylogenetic relationships among cytochrome $b$ gene sequences (HKY $+\mathrm{G}$ model) for South American camelids. Bootstrap support ( $>50 \% ; 1,000$ replicates) and Bayesian probability are indicated at nodes. Taxa membership is indicated at the right. 


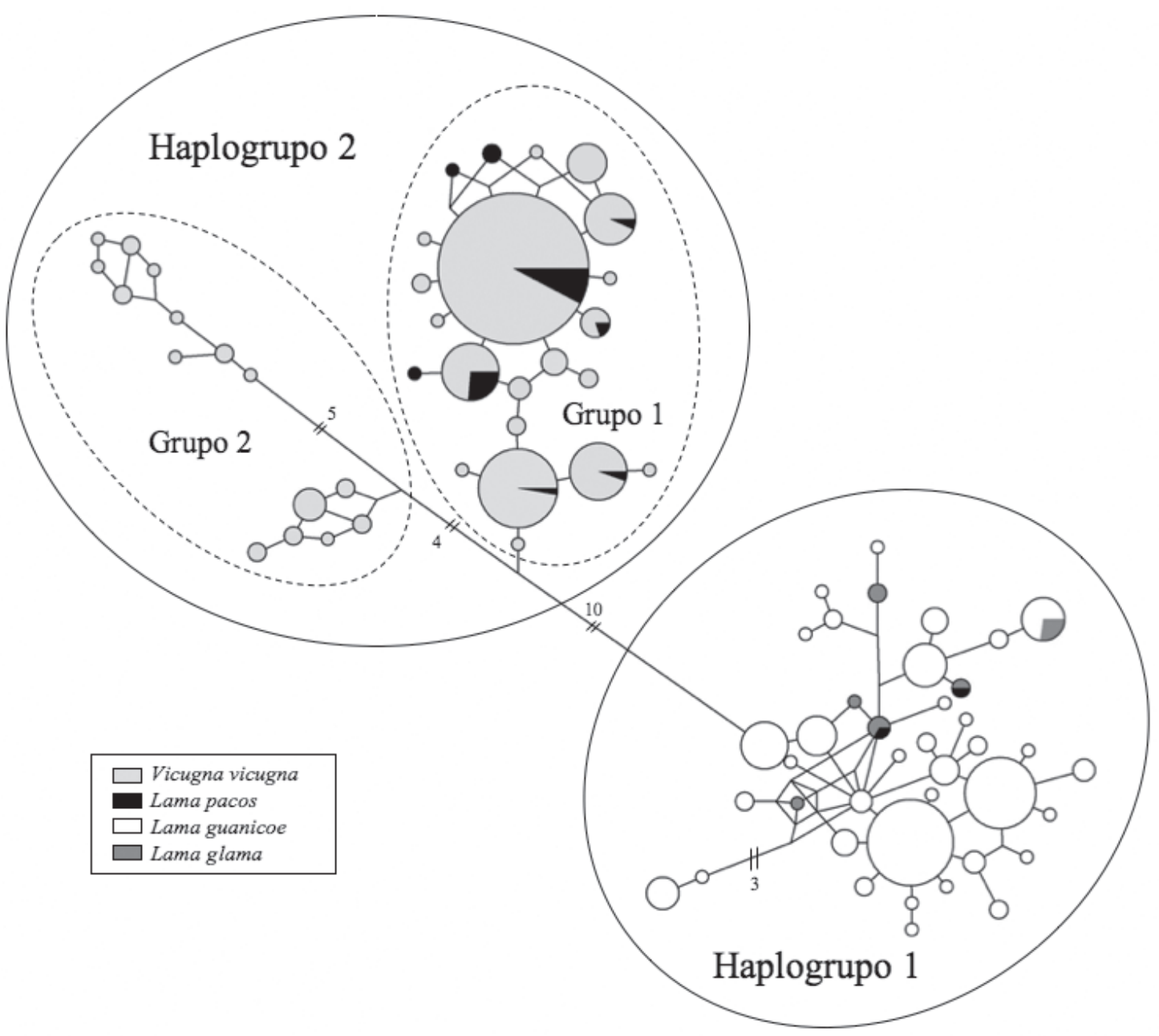

Fig. 4: Árbol de expansión mínima representando la relación entre 77 haplotipos del Dominio Hipervariable I de la Región Control. Cada haplotipo es representado por un círculo y el tamaño de ellos es proporcional a la frecuencia de cada haplotipo.

Minimun spanning network representing the relationships between 77 Hypervaraible I Domain haplotypes. Each haplotype is represented by a circle, with its size proportional to haplotype frequencies.

\section{DISCUSIÓN}

La alpaca es la especie más pequeña de los camélidos domésticos y comparte muchas características morfológicas con las vicuñas más septentrionales (Wheeler et al. 1995, Wheeler et al. 2006). Evidencia directa del proceso de domesticación está disponible en los remanentes orgánicos de camélidos sudamericanos encontrados en excavaciones arqueológicas de los Andes peruanos. Material faunístico de tales sitios arqueológicos indican que durante los inicios de la ocupación humana, tanto el guanaco, la vicuña, así como algunos cérvidos (Hippocamelus antisiensis), eran cazados persistentemente en la región (Wheeler 1984, 1995, 2000, Wing 1986). Sin embargo, en períodos posteriores (9.000-7.000 años) se registró un progresivo aumento de restos de camélidos relativo a cérvidos. Desde hace 6.000 a 5.500 años se detectan las primeras llamas y alpacas (Wheeler 1984, 1995, 2000, Wing 1986). A partir de esa fecha, restos orgánicos de camélidos neonatos comienzan a aumentar sostenidamente, hasta llegar a constituir el $70 \%$ de todos los restos de camélidos depositados (Wheeler 1995, 2000). Este aumento sugiere mortalidad debida a 
problemas epidemiológicos a causa de la domesticación (Wheeler 1995). Basado en un detallado registro dental y óseo desde uno de los sitios del Perú central, Wheeler (1991, 1995) ha colectado evidencia que concuerda con la hipótesis de que la alpaca fue domesticada a partir de poblaciones de vicuñas locales (V.v. mensalis). Finalmente, el reciente hallazgo de momias en El Yaral, MoqueguaPerú, a $1.000 \mathrm{~m}$ de altitud, no solo confirmó que la crianza de llamas y alpacas se extendía a los valles costeros, sino además, la alta selección de alpacas y llamas como productores de fibra fina (Wheeler et al. 1995).

La llama, por su parte, es el más grande de los camélidos sudamericanos (Wheeler 1995). Como el guanaco, la llama se ha adaptado a un gran rango de condiciones ambientales y la semejanza con su progenitor, L. guanicoe, es evidente en un sinnúmero de aspectos morfológicos y etológicos (Franklin 1983). Evidencias arqueozoológicas y de ADN apuntan a múltiples eventos de domesticación en el área de distribución de L.g. cacsilensis, incluyendo el Perú, posiblemente hace 6.000 años (Wheeler et al. 1976, Wing 1977, 1986, Wheeler 1984), y el norte de Chile y el noroeste de Argentina hace aproximadamente 4.400 años (Hesse 1982, Wing 1986, Mengoni Goñalons \& Yacobaccio 2006). Hace 1.400 años la crianza de llamas era establecida en la costa del Perú (Shimada \& Shimada 1985, Wheeler et al. 1995) y Ecuador (Wing 1986, Stahl 1988, Miller \& Gill 1990), llegando a jugar un importante rol en la economía local. Durante el incanato, caravanas de llamas habrían acompañado a los ejércitos reales, extendiendo su distribución a lo largo de los Andes, desde el sur de Colombia a la zona central de Chile (Wheeler 1995).

Los nuevos datos cromosómicos y moleculares aquí presentados constituyen nuevas evidencias que refuerzan una de las hipótesis previas respecto del origen de llamas y alpacas. En efecto, el hecho de que alpaca y vicuña compartan el mismo patrón de bandas $\mathrm{G}$ en el cromosoma 1 (Fig. 1 y 2), siendo diferente al que se observa tanto en guanaco como en llama, es consistente con la hipótesis de que la alpaca es un derivado de la vicuña y no del guanaco o la llama. Específicamente, sus bandas proximal oscura $1 \mathrm{p} 12$ y terminal clara 1 p13 (Fig. 2) son notoriamente diferentes a las bandas proximal clara $1 \mathrm{p} 12$ y terminal oscura 1 p13 de guanaco. Tales diferencias son observables incluso en cromosomas que provienen de la misma célula en el híbrido guanaco x alpaca (Fig. 2), lo que permite descartar que se trate de pseudodiferencias obtenidas al comparar cromosomas de distintas células. Es decir, en el híbrido F1 es posible identificar el homólogo 1 proveniente de alpaca, y separarlo del proveniente de guanaco. Adicionalmente, el hecho de que tales bandas G del cromosoma 1 de guanaco y llama sean idénticas a las observadas en camello (Fig. 1), permite postular, por el criterio extragrupo, que este estado del carácter es el primitivo, es decir, un carácter simplesiomorfo. Por lo tanto, el patrón de bandas $G$ del cromosoma 1 compartido por vicuñas y alpacas se presenta como un estado derivado, sinapomorfía exclusiva heredada a partir de un cambio producido en un ancestro común que no es ancestro de guanacos, llamas y camellos.

Los resultados obtenidos a partir del análisis del ADN mitocondrial fueron, por su parte, congruentes con lo observado en cromosomas. Con una tasa de transición-transversión más alta que la reportada para otros Artiodáctilos (Conrad et al. 1999), la topología de los árboles obtenidos mediante los tres métodos de reconstrucción filogenética (Fig. 3) reveló a vicuñas y guanacos como grupos recíprocamente monofiléticos, mostrando un solo origen para la tribu Lamini. Además, con el análisis de un mayor número de representantes del orden Artiodactyla, la tribu Camelini aparece como taxón hermano de Lamini (datos no mostrados). Sin embargo, puesto que el clado formado por las poblaciones de vicuñas más septentrionales, correspondientes a $V . v$. mensalis, incluyeron siempre a las alpacas, se pone de manifiesto la parafilia del género Lama y la incongruencia taxonómica de Lama pacos.

Un segundo resultado relevante en el linaje de vicuñas es su diferenciación en dos clados que concuerdan con la propuesta subespecífica de la especie, contrariamente a lo propuesto por Sarno et al. (2004). Un resultado similar se apreció en el árbol de expansión mínima obtenido a partir de los haplotipos del Dominio Hipervariable I (Fig. 4). Al analizar la secuencia de un gen mitocondrial con una mayor tasa de cambio, como el Dominio 
Hipervariable I de la Región Control, se observó también la formación de dos agrupamientos entre las vicuñas. Queda pendiente, sin embargo, determinar si los procesos que gobiernan la distribución geográfica y la estructura genética de las poblaciones de vicuña tienen relación con la historia evolutiva de la especie y la diferenciación subespecífica de esta.

En otro orden, la topología de los árboles obtenidos con las poblaciones de L. guanicoe reveló que los guanacos de Perú, Bolivia, Argentina y Chile forman un grupo monofilético, confirmando lo propuesto por Palma et al. (2001) donde sus ramas más basales la componen especímenes reconocidos como L. g. cacsilensis, la subespecie más septentrional del taxón (Fig. 3). Sin embargo, no es posible observar estructuración geográfica alguna entre sus representantes; de tal forma que la diferenciación de linajes no concuerda con las cuatro subespecies propuestas para esta especie. En consistencia con nuestros resultados cromosómicos, todos los árboles muestran a las llamas como parte del clado de los guanacos, mostrando a dos de sus variedades (Ccara y Chaku), como grupo hermano de los guanacos de Putre y Paposo. Paradójicamente, individuos de estas dos localidades del norte de Chile mostraron la inserción de una timina, a 22 nucleótidos del inicio de la Región Control. Puesto que esta inserción fue también detectada en el $30 \%$ de las llamas analizadas, es posible que haya ocurrido la hibridización de llamas y guanacos en esa región. Este polimorfismo también apoyaría la existencia de más de un centro de domesticación de la llama, siendo el norte de Chile uno de ellos (Grosjean et al. 1997, Messerli et al. 2000, Mengoni Goñalons \& Yacobaccio 2006). Sin embargo, es importante estudiar un número mayor de llamas, de manera de determinar la prevalencia de esta mutación en los planteles de Perú, Argentina, Chile y especialmente en Bolivia, país que alberga más de la mitad de las llamas de Sudamérica (Wheeler 1995, D. Hoces comunicación personal).

La red de haplotipos del Dominio Hipervariable I (Fig. 4) mostró dos haplogrupos diferenciados. Uno de ellos, formado por los haplotipos detectados en guanacos y llamas, revela un conflicto de participación de seis vértices, donde los haplotipos más alejados de este corresponden a guanacos cacsilensis $\mathrm{y}$ formas domésticas, tal como ocurriera en las topologías de los árboles obtenidos mediante los tres métodos de reconstrucción filogenética. Sin embargo, no es posible establecer igualmente diferencias en este haplogrupo que permitan suponer la existencia de cuatro subespecies para L. guanicoe. A 10 pasos mutacionales de ellos, las vicuñas y las alpacas revelan un segundo haplogrupo, formado por dos grupos principales que se corresponden con las subespecies $V$. v. mensalis (cluster 1), desde las cuales se derivan y comparten haplotipos las alpacas, y un segundo haplogrupo formado exclusivamente por especímenes más australes, reconocidos como V. v. vicugna (cluster 2). Todos estos resultados son congruentes con los análisis filogenéticos antes presentados y muestran el mismo esquema de diferenciación antes discutido.

La variación de un gen con una rápida tasa de cambio como el Dominio Hipervariable I, mostró también la existencia de hibridización entre especímenes domésticos, práctica muy común entre los criadores de este tipo de ganado, demostrada antes por Kadwell et al. (2001). La red de haplotipos de este gen reveló la existencia de dos haplotipos compartidos entre llamas y alpacas. Una explicación plausible para estos dos individuos con genotipo llama y fenotipo alpaca es la presencia de una madre llama en algún punto de su linaje. La ausencia, sin embargo, de haplotipos compartidos entre guanaco y alpaca, demuestra al menos preliminarmente que la hibridización ocurriría solo entre formas domésticas. A nivel andino, cuatro de cada cinco alpacas (80 \%) son híbridas (Kadwell et al. 2001). Esta elevada tasa de hibridización puede ser la causa de las disputas taxonómicas respecto del grupo, así como de la progresiva pauperización genética de las formas domésticas, hoy reflejada en el aumento del diámetro de la fibra de las alpacas actuales (Wheeler et al. 1995), en comparación con las alpacas de la preconquista.

La ausencia también de haplotipos compartidos entre guanacos y vicuñas, entre casi 500 individuos analizados, nos muestra, al igual que el análisis cromosómico, la existencia de dos linajes genéticamente bien diferenciados. Sin embargo, la ausencia de marcadores cromosómicos y moleculares 
capaces de diferenciar genéticamente una llama de un guanaco, así como una alpaca de una vicuña, pone en duda la condición de especie de llamas y alpacas, con respecto a las especies silvestres. Esta situación guarda especial relevancia cuando se tiene en cuenta que vicuñas y guanacos constituyen especies protegidas cuyas poblaciones, en algunos casos, no están del todo recuperadas (Torres 1992). Es necesario entonces contar con marcadores genéticos inequívocos, que puedan ser usados en análisis de ADN forense y permitan diferenciar especímenes domésticos de silvestres, de manera tal de ejercer un mejor control del tráfico y caza ilegal de estas especies.

En conclusión, las evidencias aquí entregadas apoyan la proposición de cambio de nombre de las alpacas a Vicugna pacos propuesta por Kadwell et al. (2001) y aceptada por Gentry et al. (2004), dada la alta similitud genética entre este taxón y las vicuñas, así como entre llamas y guanacos.

\section{AGRADECIMIENTOS}

Agradecemos el financiamiento otorgado por: CONICYT, Chile (Beca de Doctorado y Beca de Apoyo a Tesis Doctoral); FONDECYT Postdoctoral 3050046, Darwin Initiative for the Survival of Species (United Kingdom), Genetic Diversity and Management Implications for Andean Guanaco Populations in Peru, GUANACO 1, European Comission International Cooperation with Developing Countries (INCO-DC), Sustainable economic utilization of wild South American camelids: Strategies for improving rural productivity in pastoral communities in Latin America, Manejo Sostenible de Camélidos Silvestres (MACS). Los análisis cromosómicos presentados en este trabajo formaron parte de la tesis de Maestría en Producción Animal de Beatriz Zapata, desarrollada en la Pontificia Universidad Católica de Chile, con fondos del proyecto FIA - PI-C-1994-1-P-016. El trabajo de laboratorio fue realizado en el Laboratorio de Citogenética del Instituto de Nutrición y Tecnología de los Alimentos (INTA), Universidad de Chile. Agradecemos el apoyo del personal profesional y técnico del Laboratorio de Citogenética del Instituto de Nutrición y Tecnología de los
Alimentos (INTA), la colaboración del Servicio Agrícola y Ganadero (SAG) (Permiso 227, 2002) y de la Corporación Nacional Forestal (CONAF) (Autorización 6/02, 2002) por autorizar y ayudar en la captura y colecta de muestras. Agradecemos también a Patricio Hinrichsen (INIA, La Platina), Alejandra y Leonor von Baer (Criadero Llamas del Sur), al Centro de Ecología Aplicada, Minera Los Pelambres Ltda., Valchac, Luis Jacome (Zoológico de Buenos Aires, Argentina) y Alberto Duarte (Zoológico de Mendoza, Argentina) por su ayuda en la obtención de muestras. En Perú agradecemos especialmente a Carlos Loret de Mola y María Luisa del Río, y al Consejo del Medio Ambiente (CONAM); Wilder Trejo, Daniel Rivera y Daniel Arestegui, Consejo Nacional de Camélidos Sudamericanos (CONACS); Gustavo Suarez de Freitas y Antonio Morizaki, Instituto Nacional de Recursos Naturales (INRENA); Felipe San Martin, Facultad de Medicina Veterinaria, Universidad Nacional Mayor de San Marcos; y Luis Lumbreras, Instituto Nacional de Cultura (INC). También a Domingo Hoces, Katherine Yaya, Lenin Maturrano, Juan Olazabal, Hugo Castillo, Raúl Rosadio, Rocío Quispe y Jorge Rodríguez quienes contribuyeron en la presente investigación. Las muestras contaron con los permisos CITES 6282, 4222, 6007, 5971, 5177, $5178,23355,22967$ y 22920.

\section{LITERATURA CITADA}

BANDELT H J, P FORSTER \& A RÖHL (1999) Medianjoining networks for inferring intraspecific phylogenies. Molecular Biology and Evolution 16: 37-48.

BUNCH T, W FOOTE \& A MACIULIS (1985) Chromosome banding pattern homologies and NORs for the Bactrian camel, guanaco and llama. Journal of Heredity 76: 115-118.

CABRERA A (1932) Sobre los camélidos fósiles y actuales de América austral. Revista del Museo de la Plata (Argentina) 33: 89-117

CONRAD AM \& TJ ROBINSON (1999) Cytochrome b phylogeny of the family Bovidae: resolution within the Alcelaphini, Antilopini, Neotragini, and Tragelaphini. Molecular Phylogenetics and Evolution 12: 31-46.

EXCOFFIER L \& P SMOUSE (1994) Using allele frequencies and geographic subdivision to reconstruct gene trees within a species: molecular variance parsimony. Genetics 136: 343-359.

FELSENSTEIN J (1985) Confidence limits on phylogenies: an approach using the bootstrap. Evolution 39: 783-791. 
FORD E, DL POLLOCK \& G GUSTAVSON (1980) Proceedings of the First International Conference for the standardization of banded karyotypes of domestic animals. Hereditas 92: 145-162.

FRANKLIN W (1982) Biology, ecology and relationship to man of the South American camelids. En: Mares MA \& HH Genoways (eds) Mamalian biology in South America 6: 457-489. Pymatuning Laboratory of Ecology, Special Publication Series 6, University of Pittsburgh, Pittsburgh, Pennsylvania, USA.

FRANKLIN W (1983) Constrasting socioecologies of South America's wild camelids: the vicuña and the guanaco. En: Eisenberg JF \& D Kleiman (eds) Advances in the study of animal behaviour: 573629. American Society of Mammalogists, Special Publication 7, Allen Press, Lawrence, Kansas, USA.

FRISCH J L (1775) Das Natur-System der vierfüssigen Thiere. Glogau. $40 \mathrm{pp}$.

GENTRY A, J CLUTTON-BROCK \& C GROVES (2004) The naming of wild animal species and their domestic derivatives. Journal Archaeological Science 31: 645-651.

GILMORE R (1950) Fauna and ethnozoology of South Amarica. Handbook of South American indians 6: 345-464. Bureau of American Ethnography Bulletin, Washington, Smithsonian Institution. USA.

GONZÁLEZ B A, R E PALMA, B ZAPATA \& J C MARÍN (2006) Taxonomic and biogeographic status of guanaco Lama guanicoe (Artiodactyla, Camelidae). Mammal Review 36: 157-178.

GROSJEAN M, L NÚÑEZ, I CARTAJENA \& B MESSERLI (1997) Mid-Holocene climate and culture change in the Atacama desert, northern Chile. Quaternary Research 48: 239-246.

HASEGAWA M, H KISHINO \& T YANO (1985) Dating of the human-ape splitting by a molecular clock of mitochondrial DNA. Journal of Molecular Evolution 21: 160-174.

HEMMER H (1990) Domestication: the decline of environmental appreciation. Cambridge University Press, Cambridge, United Kingdom. 217 pp.

HESSE B (1982) Archaeological evidence for camelid exploitation in the Chilean Andes. Säugetierkundliche Mitteilungen 30: 201-211

HUELSENBECK J \& F RONQUIST (2001) Mr Bayes: Bayesian inference of phylogeny. Biometrics 17: 754-755.

IRWIN D, T KOCHER \& A WILSON (1991) Evolution of cytochrome $b$ gene of mammals. Journal of Molecular Evolution 32: 128-144.

KADWELL M, M FERNÁNDEZ, H STANLEY, R BALDI, JC WHEELER, R ROSADIO \& MW BRUFORD (2001) Genetic analysis reveals the wild ancestors of the llama and alpaca. Proceedings of the Royal Society of London B 268: 2575-2584.

KOULISCHER L, J TIJKENS \& J MORTELMANS (1971) The chromosomes of two male Camelidae: Camelus bactrianus and Lama vicugna. Acta Zoologica et Pathologica Antverpiensia 52: 89-92.

KRUMBIEGEL I (1944) Die neuveltlichen tylopoden. Zoologischer Anzeiger 145: 45-70.

KUMAR S, K TAMURA, I JAKOBSEN \& M NEI (2001) MEGA2: molecular evolutionary genetics analysis software, Arizona State University, Tempe, Arizona, USA.

LAHIRI DK \& JI NURNBERGER (1991) A rapid nonenzymatic method for the preparation of HMW DNA from blood for AFLP studies. Nucleic Acids Research 19: 5444
LINNAEUS C (1758) Systema Naturae per Regna tria Naturae, Secundum Classes, Ordines, Genera, Species cum Characteribus, Differentiis, Synonymis, Locis. Editio decima, reformata, Holmiae, Laurentii Salvii. 824 pp.

LÖNNBERG E (1913) Notes on guanacos. Arkiv för Zoologi 8: 1-8.

MARÍN JC (2004) Filogenia molecular, filogeografía y domesticación de camélidos sudamericanos (Artiodactyla: Camelidae). Tesis Doctoral, Facultad de Medicina, Universidad de Chile, Santiago, Chile. $159 \mathrm{pp}$.

MEDRANO JF, E AASEN \& L SHARROW (1990) DNA extraction from nucleated red blood cells. Biotechniques 8: 43.

MENGONI GOÑALONS GL \& HD YOCABACCIO (2006) The domestication of South American Camelids: A view from the south-central Andes. En: Zeder MA, DG Bradley, E Emshwiller \& B G Smith (eds) Documenting domestication: new genetic and archaeological paradigms: 228-244. University of California Press, Berkeley, California, USA.

MESSERLI B, M GROSJEAN, T HAFER, L NÚÑEZ \& C PFISTER (2000) From nature dominated to humandominated environmental changes. Quaternary Science Reviews 19: 459-479.

MILLER G \& A GILL (1990) Zooarchaelogy at Pirincay, a formative period site in highland Ecuador. Journal of Field Archaeology 17: 49-68.

MOLINA J (1782) Saggio sulla storia naturale del Chili. Tommaso d'Aquino, Bologna, Italy. 306 pp.

MÜLLER P L S (1776) Erste Classe, Säugende Thiere. In Des Ritters Carl von Linné vollständiges Naturalsystem nach der zwölften Lateinischen Ausgabe.1773-1776: pp 1-62 + 3 pls., Suppl., 384 pp., Register, 36 unnumbered pp. +536 pp.

NOWAK R (1991) Walker's mammals of the world. Fifth edition. The John Hopkins University Press, Baltimore, Maryland, USA. $1.362 \mathrm{pp}$.

OSGOOD W (1943) The mammals of Chile. Field Museum of Natural History Zoological Series 30: 1268.

PALMA RE, JC MARÍN, A SPOTORNO \& JL GALAZ (2001) Phylogenetic relationships among South American subspecies of camelids based on secuences of cytochrome b mitochondrial genes. En: Raunelli MGA (ed) Progress in South American camelids research: 44-52. Wageningen Press, Göttingen, Alemania.

PENEDO C, M FOWLER, A BOWLIN, D ANDERSON \& L GORDON (1988) Genetic variation in the blood of llamas, Lama glama, and alpacas, Lama pacos. Genetics 19: 267-276.

POSADA D \& KA CRANDALL (1998) MODELTEST: testing the model of DNA substitution. Bioinformatics 14: 817-818.

ROZAS J, JC SÁNCHEZ-DEL BARRIO, X MESSEGUER \& R ROZAS (2003) DnaSP, DNA polymorphism analyses by the coalescent and other methods. Bioinformatics 19: 2496-2497.

SAIKI R, S SCHARF, F FALOONA, K MULLIS, G HORN, H ERLICH \& N ARNHEIM (1985) Enzymatic amplification of beta-globin genomic sequences and restriction site analysis for diagnosis of sickle cell anemia. Science 230: 1350-1354

SARNO R, R HUNTER \& W FRANKLIN (1996) Immobilization of guanacos by use of tiletamine/ zolazepam. Journal of American Veterinary Medicine Association 208: 408-409. 
SARNO R, L VILLALBA, C BONACIC, B GONZÁLEZ, B ZAPATA, D MAC DONALD, S O'BRIEN \& W JOHNSON (2004) Phylogeography and subspecies assessment of vicuñas in Chile and Bolivia utilizing mtDNA and microsatellites markers; implications for vicuña conservation and management. Conservation Genetics 5: 89-102.

SCHNEIDER S, D ROESSLI \& L EXCOFFIER (2000) ARLEQUIN, Version 2000: a software for population genetics data analysis. Genetics and Biometry Laboratory, University of Geneva, Geneva, Switzerland.

SHIMADA M \& I SHIMADA (1985) Prehistoric llama breeding and herding on the north coast of Peru. American Antiquity 50: 3-26

STAHL PW (1988) Prehistoric camelids in the lowlands of western Ecuador. Journal of Archaeological Science 15: 355-365

STANLEY H, M KADWELL \& JC WHEELER (1994) Molecular evolution of the family Camelidae: a mitochondrial DNA study. Proceedings of the Royal Society of London B 256: 1-6.

SWOFFORD D (1998) PAUP (Phylogenetic Analysis Using Parsimony). Version 4.0 beta. Sinauer Associates, Sunderland, Massachusetts, USA.

TAYLOR K, D HUNGERFORD, R SNYDER \& $\mathrm{F}$ ULMER (1968) Uniformity of karyotypes in Camelidae. Cytogenetics 7: 8-15.

THOMPSON J, T GIBSON, F PLEWNIAK, F JEANMOUGIN \& D HIGGINS (1997) The ClustalX windows interface: flexible strategies for multiple sequence alignment aided by quality analysis tools. Nucleic Acids Research 24: 4876-4882.

THOMAS O (1917) Preliminary diagnosis of the new mammals obtained by the Yale National Society Peruvian Expedition. Smithsonian Miscellaneous Collection 68: 1-3.

TORRES H (1992) South American camelids: an action plan for their conservation. IUCN/SSC South American Camelids Specialist Group, Gland, Switzerland. 58 pp.

VIDAL-RIOJA L, A ZAMBELLI \& L SEMORILE (1994) An assessment of the relationships among species of Camelidae by satellite DNA comparisons. Hereditas 121: 283-290.

WALSH P, D METZGER \& R HIGUCHI (1991) Chelex ${ }^{\circledR}$ 100 as a médium for Simple Extraction of DNA for PCR-Based Typing from Forensic Material. BioTechniques 10: 506-513.
WHEELER JC (1984) On the origin and early development of camelid pastoralism in the Andes. En: Clutton-Brock J \& C Grigson (eds) Animals and archaeology. Volume 3: Early herders and their flocks: 395-410. British Archaeological Reports, BAR International Series 202, Oxford, United Kingdom.

WHEELER JC (1991) Origen, evolución y status actual. En: Fernández-Baca S (ed) Avances y perspectivas en el conocimiento de los camélidos sudamericanos: 11-48. Oficina Regional de la FAO para América Latina y el Caribe, Santiago, Chile.

WHEELER JC (1995) Evolution and present situation of the South-American Camelidae. Biological Journal of the Linnean Society 52: 271-295.

WHEELER JC (2000) Patrones prehistóricos de utilización de los camélidos sudamericanos. Boletín de Arqueología PUCP (Perú) 3: 297-306.

WHEELER JC (2005) El debate sobre el origen de la alpaca. Revista de la Academia Peruana de Ciencias Veterinarias 5-6: 40-43.

WHEELER JC, E PIRES-FERREIRA \& P KAULICKE (1976) Preceramic animal utilization in the central Peruvian Andes. Science 194: 483-490.

WHEELER JC, AJF RUSSEL \& H REDDEN (1995) Llamas and alpacas: pre-conquest breeds and postconquest hybrids. Journal of Archaeological Science 22: 833-840.

WHEELER JC, L CHIKHI \& MW BRUFORD (2006) Case study in genetics of animal domestication: South American camelids. En: Zeder MA, DG Bradley, E Emshwiller \& BG Smith (eds) Documenting domestication: new genetic and archaeological paradigms: 329-341. University of California Press, Berkeley, California, USA.

WING ES (1977) Animal domestication in the Andes. En: Reed CA (ed) Origins of agriculture: 387-859. Mouton Publishers, The Hague, The Netherlands.

WING ES (1986) Domestication of Andean mammals. En: Vuilleumier F \& M Monasterio (eds) High altitude biogeography: 246-264. Oxford University Press, Oxford, United Kingdom.

ZAPATA B (1999) Diferenciación de camélidos sudamericanos mediante análisis cariotípico. Tesis de Magíster, Facultad de Agronomía e Ingeniería Forestal, Pontificia Universidad Católica de Chile, Santiago, Chile. 140 pp. 